\title{
Algorithms for General System of Generalized Resolvent Equations with Corresponding System of Variational Inclusions
}

\author{
Lu-Chuan Ceng' and Ching-Feng Wen ${ }^{2}$ \\ ${ }^{1}$ Scientific Computing Key Laboratory of Shanghai Universities and Department of Mathematics, \\ Shanghai Normal University, Shanghai 200234, China \\ ${ }^{2}$ Center for General Education, Kaohsiung Medical University, Kaohsiung 807, Taiwan \\ Correspondence should be addressed to Ching-Feng Wen, cfwen@kmu.edu.tw
}

Received 8 January 2012; Accepted 14 January 2012

Academic Editor: Yonghong Yao

Copyright (C) 2012 L.-C. Ceng and C.-F. Wen. This is an open access article distributed under the Creative Commons Attribution License, which permits unrestricted use, distribution, and reproduction in any medium, provided the original work is properly cited.

Very recently, Ahmad and Yao (2009) introduced and considered a system of generalized resolvent equations with corresponding system of variational inclusions in uniformly smooth Banach spaces. In this paper we introduce and study a general system of generalized resolvent equations with corresponding general system of variational inclusions in uniformly smooth Banach spaces. We establish an equivalence relation between general system of generalized resolvent equations and general system of variational inclusions. The iterative algorithms for finding the approximate solutions of general system of generalized resolvent equations are proposed. The convergence criteria of approximate solutions of general system of generalized resolvent equations obtained by the proposed iterative algorithm are also presented. Our results represent the generalization, improvement, supplement, and development of Ahmad and Yao corresponding ones.

\section{Introduction and Preliminaries}

It is well known that the theory of variational inequalities has played an important role in the investigation of a wide class of problems arising in mechanics, physics, optimization and control, nonlinear programming, elasticity, and applied sciences and so on; see, for example, [1-7] and the references therein. In recent years variational inequalities have been extended and generalized in different directions. A useful and significant generalization of variational inequalities is called mixed variational inequalities involving the nonlinear term [8], which enables us to study free, moving, obstacle, equilibrium problems arising in pure and applied sciences in a unified and general framework. Due to the presence of the nonlinear term, the projection method and its variant forms including the technique of the Wiener-Hopf equations cannot be extended to suggest the iterative methods for solving 
mixed variational inequalities. To overcome these drawbacks, Hassouni and Moudafi [9] introduced variational inclusions which contain mixed variational inequalities as special cases. They studied the perturbed method for solving variational inclusions. Subsequently, M. A. Noor and K. I. Noor [10] introduced and considered the resolvent equations by virtue of the resolvent operator concept and established the equivalence between the mixed variational inequalities and the resolvent equations. The technique of resolvent equations is being used to develop powerful and efficient numerical techniques for solving mixed (quasi)variational inequalities and related optimization problems. At the same time, some iterative algorithms for approximating a solution of some system of variational inequalities are also introduced and studied in Verma [11]. Pang [12], Cohen and Chaplais [13], Binachi [14], Ansari and Yao [15] considered a system of scalar variational inequalities and Pang showed that the traffic equilibrium problem, the Nash equilibrium, and the general equilibrium programming problem can be modeled as a system of variational inequalities. As generalizations of system of variational inequalities, Agarwal et al. [16] introduced a system of generalized nonlinear mixed quasi-variational inclusions and investigated the sensitivity analysis of solutions for the system of generalized mixed quasi-variational inclusions in Hilbert spaces. In 2007, Peng and Zhu [17] considered and studied a new system of generalized mixed quasivariational inclusions with $(H, \eta)$-monotone operators and Lan et al. [18] studied a new system of nonlinear $A$-monotone multivalued variational inclusions. Furthermore, for more details in the related research work of this field, we invoke the readers to see, for instance, [19-30]. Very recently, Ahmad and Yao [31] introduced and considered a new system of variational inclusions in uniformly smooth Banach spaces, which covers the system of variational inclusions in Hilbert spaces considered by [18]. They established an equivalence relation between this system of variational inclusions and a system of generalized resolvent equations, proposed a number of iterative algorithms for this system of variational inclusions, and also gave the convergence criteria.

Let $E$ be a real Banach space with its norm $\|\cdot\|, E^{*}$ the topological dual of $E$, and $d$ the metric induced by the norm $\|\cdot\|$. Let $C B(E)$ (resp., $2^{E}$ ) be the family of all nonempty closed and bounded subsets (resp., all nonempty subsets) of $E$ and $D(\cdot, \cdot)$ the Hausdorff metric on $C B(E)$ defined by

$$
D(A, B)=\max \left\{\sup _{x \in A} d(x, B), \sup _{y \in B} d(A, y)\right\},
$$

where $d(x, B)=\inf _{y \in B} d(x, y)$ and $d(A, y)=\inf _{x \in A} d(x, y)$. We write by $J: E \rightarrow 2^{E^{*}}$ the normalized duality mapping defined as

$$
J(x)=\left\{f \in E^{*}:\langle x, f\rangle=\|x\|^{2}=\|f\|^{2}\right\}, \quad \forall x \in E,
$$

where $\langle\cdot, \cdot\rangle$ denotes the duality pairing between $E$ and $E^{*}$.

The uniform convexity of a Banach space $E$ means that for any $\epsilon>0$, there exists $\delta>0$, such that for any $x, y \in E,\|x\| \leq 1,\|y\| \leq 1,\|x-y\|=\epsilon$ ensure the following inequality:

$$
\|x+y\| \leq 2(1-\delta)
$$


The function

$$
\delta_{E}(\epsilon)=\inf \left\{1-\frac{\|x+y\|}{2}:\|x\|=1,\|y\|=1,\|x-y\|=\epsilon\right\}
$$

is called the modulus of convexity of $E$.

The uniform smoothness of a Banach space $E$ means that for any given $\epsilon>0$, there exists $\delta>0$ such that

$$
\frac{\|x+y\|+\|x-y\|}{2}-1 \leq \epsilon\|y\|
$$

holds. The function

$$
\tau_{E}(t)=\sup \left\{\frac{\|x+y\|+\|x-y\|}{2}-1:\|x\|=1,\|y\|=t\right\}
$$

is called the modulus of smoothness of $E$.

It is well known that the Banach space $E$ is uniformly convex if and only if $\delta_{E}(\epsilon)>0$ for all $\epsilon>0$, and it is uniformly smooth if and only if $\lim _{t \rightarrow 0} \tau_{E}(t) / t=0$. All Hilbert spaces, $L_{p}$ (or $\left.l_{p}\right)$ spaces $(p \geq 2)$, and the Sobolov spaces $W_{m}^{p}(p \geq 2)$ are 2-uniformly smooth, while, for $1<p \leq 2, L_{p}\left(\right.$ or $\left.l_{p}\right)$ and $W_{m}^{p}$ spaces are $p$-uniformly smooth.

Proposition 1.1 (see [15]). Let E be a uniformly smooth Banach space. Then the normalized duality mapping $J: E \rightarrow 2^{E^{*}}$ is single-valued, and for any $x, y \in E$ there holds the following:

(i) $\|x+y\|^{2} \leq\|x\|^{2}+2\langle y, J(x+y)\rangle$,

(ii) $\langle x-y, J(x)-J(y)\rangle \leq 2 C^{2} \tau_{E}(4\|x-y\| / C)$, where $C=\sqrt{\|x\|^{2}+\|y\|^{2}} / 2$.

Definition 1.2 (see [32]). A mapping $g: E \rightarrow E$ is said to be

(i) $k$-strongly accretive, $k \in(0,1)$, if for any $x, y \in E$, there exists $j(x-y) \in J(x-y)$ such that

$$
\langle g(x)-g(y), j(x-y)\rangle \geq k\|x-y\|^{2}
$$

(ii) Lipschitz continuous if for any $x, y \in E$, there exists a constant $\lambda_{g}>0$, such that

$$
\|g(x)-g(y)\| \leq \lambda_{g}\|x-y\|
$$

Definition 1.3 (see [13]). A set-valued mapping $A: E \rightarrow 2^{E}$ is said to be

(i) accretive, if for any $x, y \in E$, there exists $j(x-y) \in J(x-y)$ such that for all $u \in A(x)$ and $v \in A(y)$,

$$
\langle u-v, j(x-y)\rangle \geq 0
$$


(ii) $k$-strongly accretive, $k \in(0,1)$, if for any $x, y \in E$, there exists $j(x-y) \in J(x-y)$, such that for all $u \in A(x)$ and $v \in A(y)$,

$$
\langle u-v, j(x-y)\rangle \geq k\|x-y\|^{2}
$$

(iii) $m$-accretive if $A$ is accretive and $(I+\rho A)(E)=E$, for every (equivalently, for some) $\rho>0$, where $I$ is the identity mapping (equivalently, if $A$ is accretive and $(I+$ $A)(E)=E$ ). In particular, it is clear from [9] that if $E=H$ is a Hilbert space, than $A: E \rightarrow 2^{E}$ is an $m$-accretive mapping if and only if it is a maximal monotone mapping.

Definition 1.4 (see [31]). Let $M: E \rightarrow 2^{E}$ be an $m$-accretive mapping. For any $\rho>0$, the mapping $J_{M}^{\rho}: E \rightarrow E$ associated with $M$ defined by

$$
J_{M}^{\rho}(x)=(I+\rho M)^{-1}(x), \quad \forall x \in E
$$

is called the resolvent operator.

Definition 1.5 (see [33]). The resolvent operator $J_{M}^{\rho}: E \rightarrow E$ is said to be a retraction if

$$
(I+\rho M)^{-1} \circ(I+\rho M)^{-1}(x)=(I+\rho M)^{-1}(x), \quad \forall x \in E .
$$

It is well known that $J_{M}^{\rho}$ is a single-valued and nonexpansive mapping.

Definition 1.6 (see [10]). A set-valued mapping $H: E \rightarrow C B(E)$ is said to be $D$-Lipschitz continuous if for any $x, y \in E$, there exists a constant $\lambda_{D_{H}}>0$ such that

$$
D(H(x), H(y)) \leq \lambda_{D_{H}}\|x-y\|
$$

Let $E_{1}$ and $E_{2}$ be two real Banach spaces, $S: E_{1} \times E_{2} \rightarrow E_{1}$ and $T: E_{1} \times E_{2} \rightarrow E_{2}$ single-valued mappings, and $G: E_{1} \rightarrow 2^{E_{1}}, F: E_{2} \rightarrow 2^{E_{2}}, H: E_{1} \rightarrow 2^{E_{1}}$ and $V: E_{2} \rightarrow 2^{E_{2}}$ any four multivalued mappings. Let $M: E_{1} \rightarrow 2^{E_{1}}$ and $N: E_{2} \rightarrow 2^{E_{2}}$ be any nonlinear mappings, $m: E_{2} \rightarrow E_{1}, n: E_{1} \rightarrow E_{2}, f: E_{1} \rightarrow E_{1}$ and $g: E_{2} \rightarrow E_{2}$ nonlinear mappings with $f\left(E_{1}\right) \cap D(M) \neq \emptyset$ and $g\left(E_{2}\right) \cap D(N) \neq \emptyset$, respectively. Then we consider the problem of finding $(x, y) \in E_{1} \times E_{2},(s, v) \in G(x) \times F(y),(u, t) \in H(x) \times V(y)$ such that

$$
\begin{aligned}
& m(y) \in S(s, v)+M(f(x)), \\
& n(x) \in T(u, t)+N(g(y)),
\end{aligned}
$$

which is called a general system of variational inclusions. In particular, if $m(y)=0 \in$ $E_{1}, n(x)=0 \in E_{2}, G(x)=p(x)$ and $V(y)=q(y)$, where $p: E_{1} \rightarrow E_{1}$ and $q: E_{2} \rightarrow E_{2}$ 
are single-valued mappings, then the general system of variational inclusions (1.14) reduces to the following system of variational inclusions

$$
\begin{aligned}
& 0 \in S(p(x), v)+M(f(x)), \\
& 0 \in T(u, q(y))+N(g(y)),
\end{aligned}
$$

which was considered by Lan et al. [18] in Hilbert spaces and studied by Ahmad and Yao [31] in Banach spaces, respectively.

Proposition 1.7 (see [31, Lemma 2.1]). $(x, y) \in E_{1} \times E_{2}, u \in H(x), v \in F(y)$ is a solution of the system of variational inclusions (1.15) if and only if $(x, y, u, v)$ satisfies

$$
\begin{aligned}
& f(x)=J_{M}^{\rho}(f(x)-\rho S(p(x), v)), \quad \rho>0, \\
& g(y)=J_{N}^{\gamma}(g(y)-\gamma T(u, q(y))), \quad r>0 .
\end{aligned}
$$

Proposition 1.8 (see [31, Proposition 3.1]). The system of variational inclusions (1.15) has a solution $(x, y, u, v)$ with $(x, y) \in E_{1} \times E_{2}, u \in H(x)$ and $v \in F(y)$ if and only if the following system of generalized resolvent equations

$$
\begin{array}{ll}
S(p(x), v)+\rho^{-1} R_{M}^{\rho}\left(z^{\prime}\right)=0, & R_{M}^{\rho}=I-J_{M^{\prime}}^{\rho} \rho>0, \\
T(u, q(y))+\gamma^{-1} R_{N}^{\gamma}\left(z^{\prime \prime}\right)=0, & R_{N}^{\gamma}=I-J_{N^{\prime}}^{\gamma} \gamma>0,
\end{array}
$$

has a solution $\left(z^{\prime}, z^{\prime \prime}, x, y, u, v\right)$ with $(x, y) \in E_{1} \times E_{2}, u \in H(x), v \in F(y), z^{\prime} \in E_{1}$ and $z^{\prime \prime} \in E_{2}$, where $f(x)=J_{M}^{\rho}\left(z^{\prime}\right), g(y)=J_{N}^{\gamma}\left(z^{\prime \prime}\right)$ and $z^{\prime}=f(x)-\rho S(p(x), v), z^{\prime \prime}=g(y)-\gamma T(u, q(y))$.

Based on the above Propositions 1.7 and 1.8, Ahmad and Yao [31] presented the following algorithm and established the following strong convergence result for the sequences generated by the algorithm.

Algorithm 1.9 (see [31, Algorithm 3.1]). For given $\left(x_{0}, y_{0}\right) \in E_{1} \times E_{2}, u_{0} \in H\left(x_{0}\right), v_{0} \in$ $F\left(y_{0}\right), z_{0}^{\prime} \in E_{1}$ and $z_{0}^{\prime \prime} \in E_{2}$, compute $\left\{z_{k}^{\prime}\right\},\left\{z_{k}^{\prime \prime}\right\},\left\{x_{k}\right\},\left\{y_{k}\right\},\left\{u_{k}\right\}$, and $\left\{v_{k}\right\}$ by the iterative scheme:

$$
\begin{gathered}
f\left(x_{k}\right)=J_{M}^{\rho}\left(z_{k}^{\prime}\right), \\
g\left(y_{k}\right)=J_{N}^{\gamma}\left(z_{k}^{\prime \prime}\right), \\
u_{k} \in H\left(x_{k}\right):\left\|u_{k+1}-u_{k}\right\| \leq D\left(H\left(x_{k+1}\right), H\left(x_{k}\right)\right), \\
v_{k} \in F\left(y_{k}\right):\left\|v_{k+1}-v_{k}\right\| \leq D\left(F\left(y_{k+1}\right), F\left(y_{k}\right)\right), \\
z_{k+1}^{\prime}=f\left(x_{k}\right)-\rho S\left(p\left(x_{k}\right), v_{k}\right), \\
z_{k+1}^{\prime \prime}=g\left(y_{k}\right)-\gamma T\left(u_{k}, q\left(y_{k}\right)\right), \quad k=0,1,2, \ldots
\end{gathered}
$$

Theorem 1.10 (see [31, Theorem 3.1]). Let $E_{1}$ and $E_{2}$ be two real uniformly smooth Banach spaces with modulus of smoothness $\tau_{E_{1}}(t) \leq C_{1} t^{2}$ and $\tau_{E_{2}}(t) \leq C_{2} t^{2}$ for $C_{1}, C_{2}>0$, respectively. Let $H$ : $E_{1} \rightarrow C B\left(E_{1}\right), F: E_{2} \rightarrow C B\left(E_{2}\right)$ be D-Lipschitz continuous mappings with constants $\lambda_{D_{H}}$ and $\lambda_{D_{F}}$, respectively, and let $M: E_{1} \rightarrow 2^{E_{1}}, N: E_{2} \rightarrow 2^{E_{2}}$ be m-accretive mappings such that the 
resolvent operators associated with $M$ and $N$ are retractions. Let $f: E_{1} \rightarrow E_{1}, g: E_{2} \rightarrow E_{2}$ be both strong accretive with constants $\alpha$ and $\beta$, respectively, and Lipschitz continuous with constants $\delta_{1}$ and $\delta_{2}$, respectively. Let $p: E_{1} \rightarrow E_{1}, q: E_{2} \rightarrow E_{2}$ be Lipschitz continuous with constants $\lambda_{p}$ and $\lambda_{q}$, respectively, and let $S: E_{1} \times E_{2} \rightarrow E_{1}, T: E_{1} \times E_{2} \rightarrow E_{2}$ be Lipschitz continuous in the first and second arguments with constants $\lambda_{S_{1}}, \lambda_{S_{2}}$ and $\lambda_{T_{1}}, \lambda_{T_{2}}$, respectively.

If there exist constants $\rho>0$ and $\gamma>0$, such that

$$
\begin{aligned}
& 0<\frac{B^{\prime}+\sqrt{\theta_{1}}+\sqrt{\theta_{4}}}{1-B^{\prime}}<1, \\
& 0<\frac{B^{\prime \prime}+\sqrt{\theta_{2}}+\sqrt{\theta_{3}}}{1-B^{\prime \prime}}<1,
\end{aligned}
$$

where $B^{\prime}=\sqrt{1-2 \alpha+64 C_{1} \delta_{1}^{2}}, B^{\prime \prime}=\sqrt{1-2 \beta+64 C_{2} \delta_{2}^{2}}$ and $\theta_{1}=\left(1+\rho \lambda_{S_{1}} \lambda_{p}\right) /\left(1-\rho\left(\lambda_{S_{1}} \lambda_{p}+\right.\right.$ $\left.\left.\lambda_{S_{2}} \lambda_{D_{F}}\right)\right), \theta_{2}=\left(1+\rho \lambda_{S_{2}} \lambda_{D_{F}}\right) /\left(1-\rho\left(\lambda_{S_{1}} \lambda_{p}+\lambda_{S_{2}} \lambda_{D_{F}}\right)\right), \theta_{3}=\left(1+\gamma \lambda_{T_{2}} \lambda_{q}\right) /\left(1-\gamma\left(\lambda_{T_{1}} \lambda_{D_{H}}+\right.\right.$ $\left.\left.\lambda_{T_{2}} \lambda_{q}\right)\right), \theta_{4}=\gamma \lambda_{T_{1}} \lambda_{D_{H}} /\left(1-\gamma\left(\lambda_{T_{1}} \lambda_{D_{H}}+\lambda_{T_{2}} \lambda_{q}\right)\right)$, then there exist $(x, y) \in E_{1} \times E_{2}, u \in H(x), v \in$ $F(y)$ and $\left(z^{\prime}, z^{\prime \prime}\right) \in E_{1} \times E_{2}$ satisfying the system of generalized resolvent equations (1.17) (in this case, $(x, y, u, v)$ is a solution of system of variational inclusions (1.15)), and the iterative sequences $\left\{z_{k}^{\prime}\right\},\left\{z_{k}^{\prime \prime}\right\},\left\{x_{k}\right\},\left\{y_{k}\right\},\left\{u_{k}\right\}$, and $\left\{v_{k}\right\}$ generated by Algorithm 1.9 converge strongly to $z^{\prime}, z^{\prime \prime}, x, y, u$, and $v$, respectively.

In this paper we introduce and study a general system of generalized resolvent equations with corresponding general system of variational inclusions in uniformly smooth Banach spaces. Motivated and inspired by the above Proposition 1.8, we establish an equivalence relation between general system of generalized resolvent equations and general system of variational inclusions. By using Nadler [34] we propose some new iterative algorithms for finding the approximate solutions of general system of generalized resolvent equations, which include Ahmad and Yao's corresponding algorithms as special cases to a great extent. Furthermore, the convergence criteria of approximate solutions of general system of generalized resolvent equations obtained by the proposed iterative algorithm are also presented. There is no doubt that our results represent the generalization, improvement, supplement, and development of Ahmad and Yao corresponding ones [31].

\section{Main Results}

Let $E_{1}$ and $E_{2}$ be two real Banach spaces, let $S: E_{1} \times E_{2} \rightarrow E_{1}$ and $T: E_{1} \times E_{2} \rightarrow E_{2}$ be singlevalued mappings, and let $G: E_{1} \rightarrow 2^{E_{1}}, F: E_{2} \rightarrow 2^{E_{2}}, H: E_{1} \rightarrow 2^{E_{1}}$ and $V: E_{2} \rightarrow 2^{E_{2}}$ be any four multivalued mappings. Let $M: E_{1} \rightarrow 2^{E_{1}}$ and $N: E_{2} \rightarrow 2^{E_{2}}$ be any nonlinear mappings, $m: E_{2} \rightarrow E_{1}, n: E_{1} \rightarrow E_{2}, f: E_{1} \rightarrow E_{1}$ and $g: E_{2} \rightarrow E_{2}$ nonlinear mappings with $f\left(E_{1}\right) \cap D(M) \neq \emptyset$ and $g\left(E_{2}\right) \cap D(N) \neq \emptyset$, respectively. Then we consider the problem of finding $(x, y) \in E_{1} \times E_{2},(s, v) \in G(x) \times F(y),(u, t) \in H(x) \times V(y), z^{\prime} \in E_{1}, z^{\prime \prime} \in E_{2}$ such that

$$
\begin{aligned}
& S(s, v)+\rho^{-1} R_{M}^{\rho}\left(z^{\prime}\right)=m(y), \quad \rho>0, \\
& T(u, t)+\gamma^{-1} R_{N}^{\gamma}\left(z^{\prime \prime}\right)=n(x), \quad \gamma>0,
\end{aligned}
$$

where $R_{M}^{\rho}=I-J_{M^{\prime}}^{\rho} R_{N}^{\gamma}=I-J_{N}^{\gamma}$ and $J_{M^{\prime}}^{\rho} J_{N}^{\gamma}$ are the resolvent operators associated with $M$ and $N$, respectively. 
The corresponding general system of variational inclusions of (2.1) is the problem (1.14), that is, find $(x, y) \in E_{1} \times E_{2},(s, v) \in G(x) \times F(y),(u, t) \in H(x) \times V(y)$ such that

$$
\begin{aligned}
& m(y) \in S(s, v)+M(f(x)), \\
& n(x) \in T(u, t)+N(g(y)) .
\end{aligned}
$$

Proposition 2.1. $(x, y) \in E_{1} \times E_{2},(s, v) \in G(x) \times F(y),(u, t) \in H(x) \times V(y)$ are solutions of general system of variational inclusions (1.14) if and only if $(x, y, u, v, s, t)$ satisfies

$$
\begin{array}{ll}
f(x)=J_{M}^{\rho}[f(x)-\rho(S(s, v)-m(y))], & \rho>0, \\
g(y)=J_{N}^{\gamma}[g(y)-\gamma(T(u, t)-n(x))], & \gamma>0 .
\end{array}
$$

Proof. The proof of Proposition 2.1 is a direct consequence of the definition of resolvent operator, and hence, is omitted.

Next we first establish an equivalence relation between general system of generalized resolvent equations (2.1) and general system of variational inclusions (1.14) and then prove the existence of a solution of (2.1) and convergence of sequences generated by the proposed algorithms.

Proposition 2.2. The general system of variational inclusions (1.14) has a solution $(x, y, u, v, s, t)$ with $(x, y) \in E_{1} \times E_{2},(s, v) \in G(x) \times F(y)$ and $(u, t) \in H(x) \times V(y)$ if and only if general system of generalized resolvent equations (2.1) has a solution $\left(z^{\prime}, z^{\prime \prime}, x, y, u, v, s, t\right)$ with $(x, y) \in$ $E_{1} \times E_{2},(s, v) \in G(x) \times F(y),(u, t) \in H(x) \times V(y),\left(z^{\prime}, z^{\prime \prime}\right) \in E_{1} \times E_{2}$, where

$$
\begin{aligned}
& f(x)=J_{M}^{\rho}\left(z^{\prime}\right), \\
& g(y)=J_{N}^{\gamma}\left(z^{\prime \prime}\right),
\end{aligned}
$$

and $z^{\prime}=f(x)-\rho(S(s, v)-m(y))$ and $z^{\prime \prime}=g(y)-\gamma(T(u, t)-n(x))$.

Proof. Let $(x, y) \in E_{1} \times E_{2},(s, v) \in G(x) \times F(y),(u, t) \in H(x) \times V(y)$ be a solution of general system of variational inclusions (1.14). Then, by Proposition 2.1, it satisfies the following system of equations

$$
\begin{aligned}
& f(x)=J_{M}^{\rho}[f(x)-\rho(S(s, v)-m(y))], \\
& g(y)=J_{N}^{\gamma}[g(y)-\gamma(T(u, t)-n(x))] .
\end{aligned}
$$

Let $z^{\prime}=f(x)-\rho(S(s, v)-m(y))$ and $z^{\prime \prime}=g(y)-\gamma(T(u, t)-n(x))$. Then we have

$$
\begin{aligned}
& f(x)=J_{M}^{\rho}\left(z^{\prime}\right), \\
& g(y)=J_{N}^{\gamma}\left(z^{\prime \prime}\right),
\end{aligned}
$$


and hence $z^{\prime}=J_{M}^{\rho}\left(z^{\prime}\right)-\rho(S(s, v)-m(y))$ and $z^{\prime \prime}=J_{N}^{\gamma}\left(z^{\prime \prime}\right)-\gamma(T(u, t)-n(x))$. Thus it follows that

$$
\left(I-J_{M}^{\rho}\right)\left(z^{\prime}\right)=-\rho(S(s, v)-m(y)), \quad\left(I-J_{N}^{\gamma}\right)\left(z^{\prime \prime}\right)=-\gamma(T(u, t)-n(x)),
$$

that is,

$$
\begin{aligned}
& S(s, v)+\rho^{-1} R_{M}^{\rho}\left(z^{\prime}\right)=m(y), \\
& T(u, t)+\gamma^{-1} R_{N}^{\gamma}\left(z^{\prime \prime}\right)=n(x) .
\end{aligned}
$$

Therefore, $\left(z^{\prime}, z^{\prime \prime}, x, y, u, v, s, t\right)$ is a solution of general system of generalized resolvent equations (2.1).

Conversely, let $\left(z^{\prime}, z^{\prime \prime}, x, y, u, v, s, t\right)$ be a solution of general system of generalized resolvent equations (2.1). Then

$$
\begin{gathered}
\rho(S(s, v)-m(y))=-R_{M}^{\rho}\left(z^{\prime}\right), \\
\gamma(T(u, t)-n(x))=-R_{N}^{\gamma}\left(z^{\prime \prime}\right) .
\end{gathered}
$$

Now observe that

$$
\begin{aligned}
\rho(S(s, v)-m(y)) & =-R_{M}^{\rho}\left(z^{\prime}\right) \\
& =-\left(I-J_{M}^{\rho}\right)\left(z^{\prime}\right) \\
& =J_{M}^{\rho}\left(z^{\prime}\right)-z^{\prime} \\
& =J_{M}^{\rho}[f(x)-\rho(S(s, v)-m(y))]-[f(x)-\rho(S(s, v)-m(y))],
\end{aligned}
$$

which leads to

$$
f(x)=J_{M}^{\rho}[f(x)-\rho(S(s, v)-m(y))]
$$

and also that

$$
\begin{aligned}
r(T(u, t)-n(x)) & =-R_{N}^{\gamma}\left(z^{\prime \prime}\right) \\
& =-\left(I-J_{N}^{\gamma}\right)\left(z^{\prime \prime}\right) \\
& =J_{N}^{\gamma}\left(z^{\prime \prime}\right)-z^{\prime \prime} \\
& =J_{N}^{\gamma}[g(y)-\gamma(T(u, t)-n(x))]-[g(y)-\gamma(T(u, t)-n(x))],
\end{aligned}
$$

which leads to

$$
g(y)=J_{N}^{\gamma}[g(y)-\gamma(T(u, t)-n(x))]
$$


Consequently, we have

$$
\begin{gathered}
f(x)=J_{M}^{\rho}[f(x)-\rho(S(s, v)-m(y))], \\
g(y)=J_{N}^{\gamma}[g(y)-\gamma(T(u, t)-n(x))] .
\end{gathered}
$$

Therefore, by Proposition 2.1, $(x, y, u, v, s, t)$ is a solution of general system of variational inclusions (1.14).

Proof (Alternative). Let

$$
z^{\prime}=f(x)-\rho(S(s, v)-m(y)), \quad z^{\prime \prime}=g(y)-\gamma(T(u, t)-n(x)) .
$$

Then, utilizing (2.4), we can write

$$
z^{\prime}=J_{M}^{\rho}\left(z^{\prime}\right)-\rho(S(s, v)-m(y)), \quad z^{\prime \prime}=J_{N}^{\gamma}\left(z^{\prime \prime}\right)-\gamma(T(u, t)-n(x))
$$

which yield that

$$
\begin{aligned}
& S(s, v)+\rho^{-1} R_{M}^{\rho}\left(z^{\prime}\right)=m(y), \\
& T(u, t)+\gamma^{-1} R_{N}^{\gamma}\left(z^{\prime \prime}\right)=n(x),
\end{aligned}
$$

the required general system of generalized resolvent equations.

Algorithm 2.3. For given $\left(x_{0}, y_{0}\right) \in E_{1} \times E_{2},\left(s_{0}, v_{0}\right) \in G\left(x_{0}\right) \times F\left(y_{0}\right),\left(u_{0}, t_{0}\right) \in H\left(x_{0}\right) \times$ $V\left(y_{0}\right),\left(z_{0}^{\prime}, z_{0}^{\prime \prime}\right) \in E_{1} \times E_{2}$, compute

$$
z_{1}^{\prime}=f\left(x_{0}\right)-\rho\left(S\left(s_{0}, v_{0}\right)-m\left(y_{0}\right)\right), \quad z_{1}^{\prime \prime}=g\left(y_{0}\right)-r\left(T\left(u_{0}, t_{0}\right)-n\left(x_{0}\right)\right)
$$

For $\left(z_{1}^{\prime}, z_{1}^{\prime \prime}\right) \in E_{1} \times E_{2}$, we take $\left(x_{1}, y_{1}\right) \in E_{1} \times E_{2}$ such that $f\left(x_{1}\right)=J_{M}^{\rho}\left(z_{1}^{\prime}\right)$ and $g\left(y_{1}\right)=J_{N}^{\gamma}\left(z_{1}^{\prime \prime}\right)$. Then, by Nadler [34], there exist $\left(s_{1}, v_{1}\right) \in G\left(x_{1}\right) \times F\left(y_{1}\right),\left(u_{1}, t_{1}\right) \in H\left(x_{1}\right) \times V\left(y_{1}\right)$ such that

$$
\begin{gathered}
\left\|u_{1}-u_{0}\right\| \leq(1+1) D\left(H\left(x_{1}\right), H\left(x_{0}\right)\right) \\
\left\|v_{1}-v_{0}\right\| \leq(1+1) D\left(F\left(y_{1}\right), F\left(y_{0}\right)\right) \\
\left\|s_{1}-s_{0}\right\| \leq(1+1) D\left(G\left(x_{1}\right), G\left(x_{0}\right)\right) \\
\left\|t_{1}-t_{0}\right\| \leq(1+1) D\left(V\left(y_{1}\right), V\left(y_{0}\right)\right)
\end{gathered}
$$

where $D(\cdot, \cdot)$ is the Hausdorff metric on $C B\left(E_{1}\right)$ (for the sake of convenience, we also denote by $D(\cdot, \cdot)$ the Hausdorff metric on $\left.C B\left(E_{2}\right)\right)$. Compute

$$
z_{2}^{\prime}=f\left(x_{1}\right)-\rho\left(S\left(s_{1}, v_{1}\right)-m\left(y_{1}\right)\right), \quad z_{2}^{\prime \prime}=g\left(y_{1}\right)-r\left(T\left(u_{1}, t_{1}\right)-n\left(x_{1}\right)\right)
$$


By induction, we can obtain sequences $\left(x_{k}, y_{k}\right) \in E_{1} \times E_{2},\left(s_{k}, v_{k}\right) \in G\left(x_{k}\right) \times F\left(y_{k}\right),\left(u_{k}, t_{k}\right) \in$ $H\left(x_{k}\right) \times V\left(y_{k}\right),\left(z_{k}^{\prime}, z_{k}^{\prime \prime}\right) \in E_{1} \times E_{2}$ by the iterative scheme:

$$
\begin{gathered}
f\left(x_{k}\right)=J_{M}^{\rho}\left(z_{k}^{\prime}\right), \\
g\left(y_{k}\right)=J_{N}^{\gamma}\left(z_{k}^{\prime \prime}\right), \\
u_{k} \in H\left(x_{k}\right):\left\|u_{k+1}-u_{k}\right\| \leq\left(1+\frac{1}{k+1}\right) D\left(H\left(x_{k+1}\right), H\left(x_{k}\right)\right), \\
v_{k} \in F\left(y_{k}\right):\left\|v_{k+1}-v_{k}\right\| \leq\left(1+\frac{1}{k+1}\right) D\left(F\left(y_{k+1}\right), F\left(y_{k}\right)\right), \\
s_{k} \in G\left(x_{k}\right):\left\|s_{k+1}-s_{k}\right\| \leq\left(1+\frac{1}{k+1}\right) D\left(G\left(x_{k+1}\right), G\left(x_{k}\right)\right), \\
t_{k} \in V\left(y_{k}\right):\left\|t_{k+1}-t_{k}\right\| \leq\left(1+\frac{1}{k+1}\right) D\left(V\left(y_{k+1}\right), V\left(y_{k}\right)\right), \\
z_{k+1}^{\prime}=f\left(x_{k}\right)-\rho\left(S\left(s_{k}, v_{k}\right)-m\left(y_{k}\right)\right), \\
z_{k+1}^{\prime \prime}=g\left(y_{k}\right)-\gamma\left(T\left(u_{k}, t_{k}\right)-n\left(x_{k}\right)\right),
\end{gathered}
$$

for $k=0,1,2, \ldots$

The general system of generalized resolvent equations (2.1) can also be rewritten as

$$
\begin{aligned}
& z^{\prime}=f(x)-S(s, v)+m(y)+\left(I-\rho^{-1} R_{M}^{\rho}\right)\left(z^{\prime}\right), \\
& z^{\prime \prime}=g(y)-T(u, t)+n(x)+\left(I-\gamma^{-1} R_{N}^{\gamma}\right)\left(z^{\prime \prime}\right) .
\end{aligned}
$$

Utilizing this fixed-point formulation, we suggest the following iterative algorithm.

Algorithm 2.4. For given $\left(x_{0}, y_{0}\right) \in E_{1} \times E_{2},\left(s_{0}, v_{0}\right) \in G\left(x_{0}\right) \times F\left(y_{0}\right),\left(u_{0}, t_{0}\right) \in H\left(x_{0}\right) \times$ $V\left(y_{0}\right),\left(z_{0}^{\prime}, z_{0}^{\prime \prime}\right) \in E_{1} \times E_{2}$, compute

$$
\begin{gathered}
z_{1}^{\prime}=f\left(x_{0}\right)-S\left(s_{0}, v_{0}\right)+m\left(y_{0}\right)+\left(I-\rho^{-1} R_{M}^{\rho}\right)\left(z_{0}^{\prime}\right), \\
z_{1}^{\prime \prime}=g\left(y_{0}\right)-T\left(u_{0}, t_{0}\right)+n\left(x_{0}\right)+\left(I-\gamma^{-1} R_{N}^{\gamma}\right)\left(z_{0}^{\prime \prime}\right) .
\end{gathered}
$$

For $\left(z_{1}^{\prime}, z_{1}^{\prime \prime}\right) \in E_{1} \times E_{2}$, we take $\left(x_{1}, y_{1}\right) \in E_{1} \times E_{2}$ such that $f\left(x_{1}\right)=J_{M}^{\rho}\left(z_{1}^{\prime}\right)$ and $g\left(y_{1}\right)=J_{N}^{\gamma}\left(z_{1}^{\prime \prime}\right)$. Then, by Nadler [34], there exist $\left(s_{1}, v_{1}\right) \in G\left(x_{1}\right) \times F\left(y_{1}\right),\left(u_{1}, t_{1}\right) \in H\left(x_{1}\right) \times V\left(y_{1}\right)$ such that

$$
\begin{gathered}
\left\|u_{1}-u_{0}\right\| \leq(1+1) D\left(H\left(x_{1}\right), H\left(x_{0}\right)\right), \\
\left\|v_{1}-v_{0}\right\| \leq(1+1) D\left(F\left(y_{1}\right), F\left(y_{0}\right)\right), \\
\left\|s_{1}-s_{0}\right\| \leq(1+1) D\left(G\left(x_{1}\right), G\left(x_{0}\right)\right), \\
\left\|t_{1}-t_{0}\right\| \leq(1+1) D\left(V\left(y_{1}\right), V\left(y_{0}\right)\right),
\end{gathered}
$$


where $D(\cdot, \cdot)$ is the Hausdorff metric on $C B\left(E_{1}\right)$ (for the sake of convenience, we also denote by $D(\cdot, \cdot)$ the Hausdorff metric on $\left.C B\left(E_{2}\right)\right)$. Compute

$$
\begin{aligned}
& z_{2}^{\prime}=f\left(x_{1}\right)-S\left(s_{1}, v_{1}\right)+m\left(y_{1}\right)+\left(I-\rho^{-1} R_{M}^{\rho}\right)\left(z_{1}^{\prime}\right), \\
& z_{2}^{\prime \prime}=g\left(y_{1}\right)-T\left(u_{1}, t_{1}\right)+n\left(x_{1}\right)+\left(I-\gamma^{-1} R_{N}^{\gamma}\right)\left(z_{1}^{\prime \prime}\right) .
\end{aligned}
$$

By induction, we can obtain sequences $\left(x_{k}, y_{k}\right) \in E_{1} \times E_{2},\left(s_{k}, v_{k}\right) \in G\left(x_{k}\right) \times F\left(y_{k}\right),\left(u_{k}, t_{k}\right) \in$ $H\left(x_{k}\right) \times V\left(y_{k}\right),\left(z_{k}^{\prime}, z_{k}^{\prime \prime}\right) \in E_{1} \times E_{2}$ by the iterative scheme:

$$
\begin{gathered}
f\left(x_{k}\right)=J_{M}^{\rho}\left(z_{k}^{\prime}\right), \\
g\left(y_{k}\right)=J_{N}^{\gamma}\left(z_{k}^{\prime \prime}\right), \\
u_{k} \in H\left(x_{k}\right):\left\|u_{k+1}-u_{k}\right\| \leq\left(1+\frac{1}{k+1}\right) D\left(H\left(x_{k+1}\right), H\left(x_{k}\right)\right), \\
v_{k} \in F\left(y_{k}\right):\left\|v_{k+1}-v_{k}\right\| \leq\left(1+\frac{1}{k+1}\right) D\left(F\left(y_{k+1}\right), F\left(y_{k}\right)\right), \\
s_{k} \in G\left(x_{k}\right):\left\|s_{k+1}-s_{k}\right\| \leq\left(1+\frac{1}{k+1}\right) D\left(G\left(x_{k+1}\right), G\left(x_{k}\right)\right), \\
t_{k} \in V\left(y_{k}\right):\left\|t_{k+1}-t_{k}\right\| \leq\left(1+\frac{1}{k+1}\right) D\left(V\left(y_{k+1}\right), V\left(y_{k}\right)\right), \\
z_{k+1}^{\prime}=f\left(x_{k}\right)-S\left(s_{k}, v_{k}\right)+m\left(y_{k}\right)+\left(I-\rho^{-1} R_{M}^{\rho}\right)\left(z_{k}^{\prime}\right), \\
z_{k+1}^{\prime \prime}=g\left(y_{k}\right)-T\left(u_{k}, t_{k}\right)+n\left(x_{k}\right)+\left(I-\gamma^{-1} R_{N}^{\gamma}\right)\left(z_{k}^{\prime \prime}\right),
\end{gathered}
$$

for $k=0,1,2, \ldots$

For positive stepsize $\delta^{\prime}, \delta^{\prime \prime}$, the general system of generalized resolvent equations (2.1) can also be rewritten as

$$
\begin{aligned}
f\left(x, z^{\prime}\right) & =f\left(x, z^{\prime}\right)-\delta^{\prime}\left\{z^{\prime}-J_{M}^{\rho}\left(z^{\prime}\right)+\rho(S(s, v)-m(y))\right\} \\
& =f\left(x, z^{\prime}\right)-\delta^{\prime}\left\{f(x)-J_{M}^{\rho}(f(x))+\rho(S(s, v)-m(y))\right\}, \\
g\left(y, z^{\prime \prime}\right) & =g\left(y, z^{\prime \prime}\right)-\delta^{\prime \prime}\left\{z^{\prime \prime}-J_{N}^{\gamma}\left(z^{\prime \prime}\right)+\gamma(T(u, t)-n(x))\right\} \\
& =g\left(y, z^{\prime \prime}\right)-\delta^{\prime \prime}\left\{g(y)-J_{N}^{\gamma}(g(y))+\gamma(T(u, t)-n(x))\right\} .
\end{aligned}
$$

This fixed point formulation enables us to propose the following iterative algorithm.

Algorithm 2.5. For given $\left(x_{0}, y_{0}\right) \in E_{1} \times E_{2},\left(s_{0}, v_{0}\right) \in G\left(x_{0}\right) \times F\left(y_{0}\right),\left(u_{0}, t_{0}\right) \in H\left(x_{0}\right) \times$ $V\left(y_{0}\right),\left(z_{0}^{\prime}, z_{0}^{\prime \prime}\right) \in E_{1} \times E_{2}$, compute $\left(x_{1}, y_{1}\right) \in E_{1} \times E_{2}$ and $\left(z_{1}^{\prime}, z_{1}^{\prime \prime}\right) \in E_{1} \times E_{2}$ such that

$$
\begin{aligned}
& f\left(x_{1}, z_{1}^{\prime}\right)=f\left(x_{0}, z_{0}^{\prime}\right)-\delta^{\prime}\left\{f\left(x_{0}\right)-J_{M}^{\rho}\left(f\left(x_{0}\right)\right)+\rho\left(S\left(s_{0}, v_{0}\right)-m\left(y_{0}\right)\right)\right\}, \\
& g\left(y_{1}, z_{1}^{\prime \prime}\right)=g\left(y_{0}, z_{0}^{\prime \prime}\right)-\delta^{\prime \prime}\left\{g\left(y_{0}\right)-J_{N}^{\gamma}\left(g\left(y_{0}\right)\right)+\gamma\left(T\left(u_{0}, t_{0}\right)-n\left(x_{0}\right)\right)\right\} .
\end{aligned}
$$


Then, by Nadler [34], there exist $\left(s_{1}, v_{1}\right) \in G\left(x_{1}\right) \times F\left(y_{1}\right),\left(u_{1}, t_{1}\right) \in H\left(x_{1}\right) \times V\left(y_{1}\right)$ such that

$$
\begin{gathered}
\left\|u_{1}-u_{0}\right\| \leq(1+1) D\left(H\left(x_{1}\right), H\left(x_{0}\right)\right) \\
\left\|v_{1}-v_{0}\right\| \leq(1+1) D\left(F\left(y_{1}\right), F\left(y_{0}\right)\right) \\
\left\|s_{1}-s_{0}\right\| \leq(1+1) D\left(G\left(x_{1}\right), G\left(x_{0}\right)\right) \\
\left\|t_{1}-t_{0}\right\| \leq(1+1) D\left(V\left(y_{1}\right), V\left(y_{0}\right)\right)
\end{gathered}
$$

where $D(\cdot, \cdot)$ is the Hausdorff metric on $C B\left(E_{1}\right)$ (for the sake of convenience, we also denote by $D(\cdot, \cdot)$ the Hausdorff metric on $\left.C B\left(E_{2}\right)\right)$. Compute $\left(x_{2}, y_{2}\right) \in E_{1} \times E_{2}$ and $\left(z_{2}^{\prime}, z_{2}^{\prime \prime}\right) \in E_{1} \times E_{2}$ such that

$$
\begin{aligned}
& f\left(x_{2}, z_{2}^{\prime}\right)=f\left(x_{1}, z_{1}^{\prime}\right)-\delta^{\prime}\left\{f\left(x_{1}\right)-J_{M}^{\rho}\left(f\left(x_{1}\right)\right)+\rho\left(S\left(s_{1}, v_{1}\right)-m\left(y_{1}\right)\right)\right\} \\
& g\left(y_{2}, z_{2}^{\prime \prime}\right)=g\left(y_{1}, z_{1}^{\prime \prime}\right)-\delta^{\prime \prime}\left\{g\left(y_{1}\right)-J_{N}^{\gamma}\left(g\left(y_{1}\right)\right)+\gamma\left(T\left(u_{1}, t_{1}\right)-n\left(x_{1}\right)\right)\right\} .
\end{aligned}
$$

By induction, we can obtain sequences $\left(x_{k}, y_{k}\right) \in E_{1} \times E_{2},\left(s_{k}, v_{k}\right) \in G\left(x_{k}\right) \times F\left(y_{k}\right),\left(u_{k}, t_{k}\right) \in$ $H\left(x_{k}\right) \times V\left(y_{k}\right),\left(z_{k}^{\prime}, z_{k}^{\prime \prime}\right) \in E_{1} \times E_{2}$ by the iterative scheme:

$$
\begin{gathered}
u_{k} \in H\left(x_{k}\right):\left\|u_{k+1}-u_{k}\right\| \leq\left(1+\frac{1}{k+1}\right) D\left(H\left(x_{k+1}\right), H\left(x_{k}\right)\right), \\
v_{k} \in F\left(y_{k}\right):\left\|v_{k+1}-v_{k}\right\| \leq\left(1+\frac{1}{k+1}\right) D\left(F\left(y_{k+1}\right), F\left(y_{k}\right)\right), \\
s_{k} \in G\left(x_{k}\right):\left\|s_{k+1}-s_{k}\right\| \leq\left(1+\frac{1}{k+1}\right) D\left(G\left(x_{k+1}\right), G\left(x_{k}\right)\right), \\
t_{k} \in V\left(y_{k}\right):\left\|t_{k+1}-t_{k}\right\| \leq\left(1+\frac{1}{k+1}\right) D\left(V\left(y_{k+1}\right), V\left(y_{k}\right)\right), \\
f\left(x_{k+1}, z_{k+1}^{\prime}\right)=f\left(x_{k}, z_{k}^{\prime}\right)-\delta^{\prime}\left\{f\left(x_{k}\right)-J_{M}^{\rho}\left(f\left(x_{k}\right)\right)+\rho\left(S\left(s_{k}, v_{k}\right)-m\left(y_{k}\right)\right)\right\}, \\
g\left(y_{k+1}, z_{k+1}^{\prime \prime}\right)=g\left(y_{k}, z_{k}^{\prime \prime}\right)-\delta^{\prime \prime}\left\{g\left(y_{k}\right)-J_{N}^{\gamma}\left(g\left(y_{k}\right)\right)+\gamma\left(T\left(u_{k}, t_{k}\right)-n\left(x_{k}\right)\right)\right\},
\end{gathered}
$$

for $k=0,1,2, \ldots$

Note that for $\delta^{\prime}=\delta^{\prime \prime}=1, f\left(x_{k}, z_{k}^{\prime}\right)=f\left(x_{k}\right), g\left(y_{k}, z_{k}^{\prime \prime}\right)=g\left(y_{k}\right)$, Algorithm 2.5 reduces to the following algorithm which solves the general system of variational inclusions (1.14).

Algorithm 2.6. For given $\left(x_{0}, y_{0}\right) \in E_{1} \times E_{2},\left(s_{0}, v_{0}\right) \in G\left(x_{0}\right) \times F\left(y_{0}\right),\left(u_{0}, t_{0}\right) \in H\left(x_{0}\right) \times V\left(y_{0}\right)$, compute $\left(x_{1}, y_{1}\right) \in E_{1} \times E_{2}$ such that

$$
\begin{gathered}
f\left(x_{1}\right)=J_{M}^{\rho}\left[f\left(x_{0}\right)-\rho\left(S\left(s_{0}, v_{0}\right)-m\left(y_{0}\right)\right)\right], \\
g\left(y_{1}\right)=J_{N}^{\gamma}\left[g\left(y_{0}\right)-\gamma\left(T\left(u_{0}, t_{0}\right)-n\left(x_{0}\right)\right)\right] .
\end{gathered}
$$


Then, by Nadler [34], there exist $\left(s_{1}, v_{1}\right) \in G\left(x_{1}\right) \times F\left(y_{1}\right),\left(u_{1}, t_{1}\right) \in H\left(x_{1}\right) \times V\left(y_{1}\right)$ such that

$$
\begin{gathered}
\left\|u_{1}-u_{0}\right\| \leq(1+1) D\left(H\left(x_{1}\right), H\left(x_{0}\right)\right), \\
\left\|v_{1}-v_{0}\right\| \leq(1+1) D\left(F\left(y_{1}\right), F\left(y_{0}\right)\right), \\
\left\|s_{1}-s_{0}\right\| \leq(1+1) D\left(G\left(x_{1}\right), G\left(x_{0}\right)\right), \\
\left\|t_{1}-t_{0}\right\| \leq(1+1) D\left(V\left(y_{1}\right), V\left(y_{0}\right)\right),
\end{gathered}
$$

where $D(\cdot, \cdot)$ is the Hausdorff metric on $C B\left(E_{1}\right)$ (for the sake of convenience, we also denote by $D(\cdot, \cdot)$ the Hausdorff metric on $\left.C B\left(E_{2}\right)\right)$. Compute $\left(x_{2}, y_{2}\right) \in E_{1} \times E_{2}$ such that

$$
\begin{aligned}
& f\left(x_{2}\right)=J_{M}^{\rho}\left[f\left(x_{1}\right)-\rho\left(S\left(s_{1}, v_{1}\right)-m\left(y_{1}\right)\right)\right], \\
& g\left(y_{2}\right)=J_{N}^{\gamma}\left[g\left(y_{1}\right)-\gamma\left(T\left(u_{1}, t_{1}\right)-n\left(x_{1}\right)\right)\right] .
\end{aligned}
$$

By induction, we can obtain sequences $\left(x_{k}, y_{k}\right) \in E_{1} \times E_{2},\left(s_{k}, v_{k}\right) \in G\left(x_{k}\right) \times F\left(y_{k}\right),\left(u_{k}, t_{k}\right) \in$ $H\left(x_{k}\right) \times V\left(y_{k}\right)$ by the iterative scheme:

$$
\begin{gathered}
f\left(x_{k+1}\right)=J_{M}^{\rho}\left[f\left(x_{k}\right)-\rho\left(S\left(s_{k}, v_{k}\right)-m\left(y_{k}\right)\right)\right], \\
g\left(y_{k+1}\right)=J_{N}^{\gamma}\left[g\left(y_{k}\right)-\gamma\left(T\left(u_{k}, t_{k}\right)-n\left(x_{k}\right)\right)\right], \\
u_{k} \in H\left(x_{k}\right):\left\|u_{k+1}-u_{k}\right\| \leq\left(1+\frac{1}{k+1}\right) D\left(H\left(x_{k+1}\right), H\left(x_{k}\right)\right), \\
v_{k} \in F\left(y_{k}\right):\left\|v_{k+1}-v_{k}\right\| \leq\left(1+\frac{1}{k+1}\right) D\left(F\left(y_{k+1}\right), F\left(y_{k}\right)\right), \\
s_{k} \in G\left(x_{k}\right):\left\|s_{k+1}-s_{k}\right\| \leq\left(1+\frac{1}{k+1}\right) D\left(G\left(x_{k+1}\right), G\left(x_{k}\right)\right), \\
t_{k} \in V\left(y_{k}\right):\left\|t_{k+1}-t_{k}\right\| \leq\left(1+\frac{1}{k+1}\right) D\left(V\left(y_{k+1}\right), V\left(y_{k}\right)\right),
\end{gathered}
$$

for $k=0,1,2, \ldots$

We now study the convergence analysis of Algorithm 2.3. In a similar way, one can study the convergence of other algorithms.

Theorem 2.7. Let $E_{1}$ and $E_{2}$ be two real uniformly smooth Banach spaces with modulus of smoothness $\tau_{E_{1}}(t) \leq C_{1} t^{2}$ and $\tau_{E_{2}}(t) \leq C_{2} t^{2}$ for $C_{1}, C_{2}>0$, respectively. Let $G: E_{1} \rightarrow C B\left(E_{1}\right), F: E_{2} \rightarrow$ $C B\left(E_{2}\right), H: E_{1} \rightarrow C B\left(E_{1}\right), V: E_{2} \rightarrow C B\left(E_{2}\right)$ be D-Lipschitz continuous mappings with constants $\lambda_{D_{G}}, \lambda_{D_{F}}, \lambda_{D_{H}}$, and $\lambda_{D_{V}}$, respectively, and let $M: E_{1} \rightarrow 2^{E_{1}}, N: E_{2} \rightarrow 2^{E_{2}}$ be maccretive mappings such that the resolvent operators associated with $M$ and $N$ are retractions. Let $f: E_{1} \rightarrow E_{1}, g: E_{2} \rightarrow E_{2}$ be both strong accretive with constants $\alpha$ and $\beta$, respectively, and Lipschitz continuous with constants $\delta_{1}$ and $\delta_{2}$, respectively. Let $m: E_{2} \rightarrow E_{1}, n: E_{1} \rightarrow E_{2}$ be Lipschitz continuous with constants $\lambda_{m}$ and $\lambda_{n}$, respectively, and $S: E_{1} \times E_{2} \rightarrow E_{1}, T$ : $E_{1} \times E_{2} \rightarrow E_{2}$ Lipschitz continuous in the first and second arguments with constants $\lambda_{S_{1}}, \lambda_{S_{2}}$ and $\lambda_{T_{1}}, \lambda_{T_{2}}$, respectively. 
If there exist constants $\rho>0$ and $\gamma>0$, such that

$$
\begin{aligned}
& 0<\frac{B^{\prime}+\sqrt{\theta_{1}}+\sqrt{\theta_{4}}}{1-B^{\prime}}<1, \\
& 0<\frac{B^{\prime \prime}+\sqrt{\theta_{2}}+\sqrt{\theta_{3}}}{1-B^{\prime \prime}}<1,
\end{aligned}
$$

where $B^{\prime}=\sqrt{1-2 \alpha+64 C_{1} \delta_{1}^{2}}, B^{\prime \prime}=\sqrt{1-2 \beta+64 C_{2} \delta_{2}^{2}}$, and $\theta_{1}=\left(1+\rho \lambda_{S_{1}} \lambda_{D_{G}}\right) /\left(1-\rho\left(\lambda_{S_{1}} \lambda_{D_{G}}+\right.\right.$ $\left.\left.\lambda_{S_{2}} \lambda_{D_{F}}+\lambda_{m}\right)\right), \theta_{2}=\rho\left(\lambda_{S_{2}} \lambda_{D_{F}}+\lambda_{m}\right) /\left(1-\rho\left(\lambda_{S_{1}} \lambda_{D_{G}}+\lambda_{S_{2}} \lambda_{D_{F}}+\lambda_{m}\right)\right), \theta_{3}=\left(1+\gamma \lambda_{T_{2}} \lambda_{D_{V}}\right) /(1-$ $\left.\gamma\left(\lambda_{T_{1}} \lambda_{D_{H}}+\lambda_{T_{2}} \lambda_{D_{V}}+\lambda_{n}\right)\right), \theta_{4}=\gamma\left(\lambda_{T_{1}} \lambda_{D_{H}}+\lambda_{n}\right) /\left(1-\gamma\left(\lambda_{T_{1}} \lambda_{D_{H}}+\lambda_{T_{2}} \lambda_{D_{V}}+\lambda_{n}\right)\right)$, then there exist $(x, y) \in E_{1} \times E_{2},(s, v) \in G(x) \times F(y),(u, t) \in H(x) \times V(y)$ and $\left(z^{\prime}, z^{\prime \prime}\right) \in E_{1} \times E_{2}$ satisfying the general system of generalized resolvent equations (2.1) (in this case, $(x, y, u, v, s, t)$ is a solution of general system of variational inclusions (1.14)), and the iterative sequences $\left\{z_{k}^{\prime}\right\},\left\{z_{k}^{\prime \prime}\right\},\left\{x_{k}\right\},\left\{y_{k}\right\}$, $\left\{u_{k}\right\},\left\{v_{k}\right\},\left\{s_{k}\right\}$, and $\left\{t_{k}\right\}$ generated by Algorithm 2.3 converge strongly to $z^{\prime}, z^{\prime \prime}, x, y, u, v, s$, and $t$, respectively.

Proof. From Algorithm 2.3 we have

$$
\begin{aligned}
\left\|z_{k+1}^{\prime}-z_{k}^{\prime}\right\|= & \left\|f\left(x_{k}\right)-\rho\left(S\left(s_{k}, v_{k}\right)-m\left(y_{k}\right)\right)-\left[f\left(x_{k-1}\right)-\rho\left(S\left(s_{k-1}, v_{k-1}\right)-m\left(y_{k-1}\right)\right)\right]\right\| \\
\leq & \left\|x_{k}-x_{k-1}-\left(f\left(x_{k}\right)-f\left(x_{k-1}\right)\right)\right\| \\
& \quad+\left\|x_{k}-x_{k-1}-\rho\left[\left(S\left(s_{k}, v_{k}\right)-m\left(y_{k}\right)\right)-\left(S\left(s_{k-1}, v_{k-1}\right)-m\left(y_{k-1}\right)\right)\right]\right\| .
\end{aligned}
$$

By Proposition 1.1, we have (see, e.g., the proof of [32, Theorem 3])

$$
\left\|x_{k}-x_{k-1}-\left(f\left(x_{k}\right)-f\left(x_{k-1}\right)\right)\right\|^{2} \leq\left(1-2 \alpha+64 C \delta_{1}^{2}\right)\left\|x_{k}-x_{k-1}\right\|^{2} .
$$

Since $S$ is Lipschitz continuous in both arguments, $G, F$ are $D$-Lipschitz continuous, and $m$ is Lipschitz continuous, we have

$$
\begin{aligned}
\left\|\left(S\left(s_{k}, v_{k}\right)-m\left(y_{k}\right)\right)-\left(S\left(s_{k-1}, v_{k-1}\right)-m\left(y_{k-1}\right)\right)\right\| \\
\quad=\left\|S\left(s_{k}, v_{k}\right)-S\left(s_{k-1}, v_{k}\right)+S\left(s_{k-1}, v_{k}\right)-S\left(s_{k-1}, v_{k-1}\right)-\left(m\left(y_{k}\right)-m\left(y_{k-1}\right)\right)\right\| \\
\quad \leq\left\|S\left(s_{k}, v_{k}\right)-S\left(s_{k-1}, v_{k}\right)\right\|+\left\|S\left(s_{k-1}, v_{k}\right)-S\left(s_{k-1}, v_{k-1}\right)\right\|+\left\|m\left(y_{k}\right)-m\left(y_{k-1}\right)\right\| \\
\quad \leq \lambda_{S_{1}}\left\|s_{k}-s_{k-1}\right\|+\lambda_{S_{2}}\left\|v_{k}-v_{k-1}\right\|+\lambda_{m}\left\|y_{k}-y_{k-1}\right\| \\
\quad \leq \lambda_{S_{1}}\left(1+\frac{1}{k}\right) D\left(G\left(x_{k}\right), G\left(x_{k-1}\right)\right)+\lambda_{S_{2}}\left(1+\frac{1}{k}\right) D\left(F\left(y_{k}\right), F\left(y_{k-1}\right)\right)+\lambda_{m}\left\|y_{k}-y_{k-1}\right\| \\
\quad \leq\left(1+\frac{1}{k}\right) \lambda_{S_{1}} \lambda_{D_{G}}\left\|x_{k}-x_{k-1}\right\|+\left(1+\frac{1}{k}\right) \lambda_{S_{2}} \lambda_{D_{F}}\left\|y_{k}-y_{k-1}\right\|+\lambda_{m}\left\|y_{k}-y_{k-1}\right\| \\
\quad=\left(1+\frac{1}{k}\right) \lambda_{S_{1}} \lambda_{D_{G}}\left\|x_{k}-x_{k-1}\right\|+\left[\left(1+\frac{1}{k}\right) \lambda_{S_{2}} \lambda_{D_{F}}+\lambda_{m}\right]\left\|y_{k}-y_{k-1}\right\| .
\end{aligned}
$$


Utilizing (2.41) and Proposition 1.1, we have

$$
\begin{aligned}
&\left\|x_{k}-x_{k-1}-\rho\left[\left(S\left(s_{k}, v_{k}\right)-m\left(y_{k}\right)\right)-\left(S\left(s_{k-1}, v_{k-1}\right)-m\left(y_{k-1}\right)\right)\right]\right\|^{2} \\
& \leq\left\|x_{k}-x_{k-1}\right\|^{2}-2 \rho\left\langle\left(S\left(s_{k}, v_{k}\right)-m\left(y_{k}\right)\right)-\left(S\left(s_{k-1}, v_{k-1}\right)-m\left(y_{k-1}\right)\right),\right. \\
&\left.\quad J\left(x_{k}-x_{k-1}-\rho\left[\left(S\left(s_{k}, v_{k}\right)-m\left(y_{k}\right)\right)-\left(S\left(s_{k-1}, v_{k-1}\right)-m\left(y_{k-1}\right)\right)\right]\right)\right\rangle \\
& \leq\left\|x_{k}-x_{k-1}\right\|^{2}+2 \rho\left\|\left(S\left(s_{k}, v_{k}\right)-m\left(y_{k}\right)\right)-\left(S\left(s_{k-1}, v_{k-1}\right)-m\left(y_{k-1}\right)\right)\right\| \\
& \times\left\|x_{k}-x_{k-1}-\rho\left[\left(S\left(s_{k}, v_{k}\right)-m\left(y_{k}\right)\right)-\left(S\left(s_{k-1}, v_{k-1}\right)-m\left(y_{k-1}\right)\right)\right]\right\| \\
& \leq\left\|x_{k}-x_{k-1}\right\|^{2}+2 \rho\left\{\left(1+\frac{1}{k}\right) \lambda_{S_{1}} \lambda_{D_{G}}\left\|x_{k}-x_{k-1}\right\|+\left[\left(1+\frac{1}{k}\right) \lambda_{S_{2}} \lambda_{D_{F}}+\lambda_{m}\right]\left\|y_{k}-y_{k-1}\right\|\right\} \\
& \times\left\|x_{k}-x_{k-1}-\rho\left[\left(S\left(s_{k}, v_{k}\right)-m\left(y_{k}\right)\right)-\left(S\left(s_{k-1}, v_{k-1}\right)-m\left(y_{k-1}\right)\right)\right]\right\| \\
& \leq\left\|x_{k}-x_{k-1}\right\|^{2}+\rho\left(1+\frac{1}{k}\right) \lambda_{S_{1}} \lambda_{D_{G}} \\
& \times\left\{\left\|x_{k}-x_{k-1}\right\|^{2}+\left\|x_{k}-x_{k-1}-\rho\left[\left(S\left(s_{k}, v_{k}\right)-m\left(y_{k}\right)\right)-\left(S\left(s_{k-1}, v_{k-1}\right)-m\left(y_{k-1}\right)\right)\right]\right\|^{2}\right\} \\
&+\rho\left[\left(1+\frac{1}{k}\right) \lambda_{S_{2}} \lambda_{D_{F}}+\lambda_{m}\right] \\
& \times\left\{\left\|y_{k}-y_{k-1}\right\|^{2}+\left\|x_{k}-x_{k-1}-\rho\left[\left(S\left(s_{k}, v_{k}\right)-m\left(y_{k}\right)\right)-\left(S\left(s_{k-1}, v_{k-1}\right)-m\left(y_{k-1}\right)\right)\right]\right\|^{2}\right\} \\
&= {\left[1+\rho\left(1+\frac{1}{k}\right) \lambda_{S_{1}} \lambda_{D_{G}}\right]\left\|x_{k}-x_{k-1}\right\|^{2}+\rho\left[\left(1+\frac{1}{k}\right)\left(\lambda_{S_{1}} \lambda_{D_{G}}+\lambda_{S_{2}} \lambda_{D_{F}}\right)+\lambda_{m}\right] } \\
& \times\left\|x_{k}-x_{k-1}-\rho\left[\left(S\left(s_{k}, v_{k}\right)-m\left(y_{k}\right)\right)-\left(S\left(s_{k-1}, v_{k-1}\right)-m\left(y_{k-1}\right)\right)\right]\right\|^{2} \\
&+\rho\left[\left(1+\frac{1}{k}\right) \lambda_{S_{2}} \lambda_{D_{F}}+\lambda_{m}\right]\left\|y_{k}-y_{k-1}\right\|^{2},
\end{aligned}
$$

which implies that

$$
\begin{aligned}
\| x_{k}- & x_{k-1}-\rho\left[\left(S\left(s_{k}, v_{k}\right)-m\left(y_{k}\right)\right)-\left(S\left(s_{k-1}, v_{k-1}\right)-m\left(y_{k-1}\right)\right)\right] \|^{2} \\
\leq & \frac{1+\rho(1+1 / k) \lambda_{S_{1}} \lambda_{D_{G}}}{1-\rho\left[(1+1 / k)\left(\lambda_{S_{1}} \lambda_{D_{G}}+\lambda_{S_{2}} \lambda_{D_{F}}\right)+\lambda_{m}\right]}\left\|x_{k}-x_{k-1}\right\|^{2} \\
& +\frac{\rho\left[(1+1 / k) \lambda_{S_{2}} \lambda_{D_{F}}+\lambda_{m}\right]}{1-\rho\left[(1+1 / k)\left(\lambda_{S_{1}} \lambda_{D_{G}}+\lambda_{S_{2}} \lambda_{D_{F}}\right)+\lambda_{m}\right]}\left\|y_{k}-y_{k-1}\right\|^{2} \\
\leq & \frac{1+\rho(1+1 / k) \lambda_{S_{1}} \lambda_{D_{G}}}{1-\rho\left[(1+1 / k)\left(\lambda_{S_{1}} \lambda_{D_{G}}+\lambda_{S_{2}} \lambda_{D_{F}}\right)+\lambda_{m}\right]}\left\|x_{k}-x_{k-1}\right\|^{2} \\
& +\frac{\rho\left[(1+1 / k) \lambda_{S_{2}} \lambda_{D_{F}}+\lambda_{m}\right]}{1-\rho\left[(1+1 / k)\left(\lambda_{S_{1}} \lambda_{D_{G}}+\lambda_{S_{2}} \lambda_{D_{F}}\right)+\lambda_{m}\right]}\left\|y_{k}-y_{k-1}\right\|^{2} \\
& +2 \sqrt{\frac{1+\rho(1+1 / k) \lambda_{S_{1}} \lambda_{D_{G}}}{1-\rho\left[(1+1 / k)\left(\lambda_{S_{1}} \lambda_{D_{G}}+\lambda_{S_{2}} \lambda_{D_{F}}\right)+\lambda_{m}\right]}} \sqrt{\frac{\rho\left[(1+1 / k) \lambda_{S_{2}} \lambda_{D_{F}}+\lambda_{m}\right]}{1-\rho\left[(1+1 / k)\left(\lambda_{S_{1}} \lambda_{D_{G}}+\lambda_{S_{2}} \lambda_{D_{F}}\right)+\lambda_{m}\right]}} \\
& \times\left\|x_{k}-x_{k-1}\right\|\left\|y_{k}-y_{k-1}\right\|
\end{aligned}
$$




$$
\begin{aligned}
& =\left\{\sqrt{\frac{1+\rho(1+1 / k) \lambda_{S_{1}} \lambda_{D_{G}}}{1-\rho\left[(1+1 / k)\left(\lambda_{S_{1}} \lambda_{D_{G}}+\lambda_{S_{2}} \lambda_{D_{F}}\right)+\lambda_{m}\right]}}\left\|x_{k}-x_{k-1}\right\|\right. \\
& \left.+\sqrt{\frac{\rho\left[(1+1 / k) \lambda_{S_{2}} \lambda_{D_{F}}+\lambda_{m}\right]}{1-\rho\left[(1+1 / k)\left(\lambda_{S_{1}} \lambda_{D_{G}}+\lambda_{S_{2}} \lambda_{D_{F}}\right)+\lambda_{m}\right]}}\left\|y_{k}-y_{k-1}\right\|\right\}^{2} .
\end{aligned}
$$

Thus, we have

$$
\begin{aligned}
& \left\|x_{k}-x_{k-1}-\rho\left[\left(S\left(s_{k}, v_{k}\right)-m\left(y_{k}\right)\right)-\left(S\left(s_{k-1}, v_{k-1}\right)-m\left(y_{k-1}\right)\right)\right]\right\| \\
& \leq \sqrt{\frac{1+\rho(1+1 / k) \lambda_{S_{1}} \lambda_{D_{G}}}{1-\rho\left[(1+1 / k)\left(\lambda_{S_{1}} \lambda_{D_{G}}+\lambda_{S_{2}} \lambda_{D_{F}}\right)+\lambda_{m}\right]}}\left\|x_{k}-x_{k-1}\right\| \\
& \quad+\sqrt{\frac{\rho\left[(1+1 / k) \lambda_{S_{2}} \lambda_{D_{F}}+\lambda_{m}\right]}{1-\rho\left[(1+1 / k)\left(\lambda_{S_{1}} \lambda_{D_{G}}+\lambda_{S_{2}} \lambda_{D_{F}}\right)+\lambda_{m}\right]}}\left\|y_{k}-y_{k-1}\right\| .
\end{aligned}
$$

Note that

$$
\begin{aligned}
& \lim _{k \rightarrow \infty} \frac{1+\rho(1+1 / k) \lambda_{S_{1}} \lambda_{D_{G}}}{1-\rho\left[(1+1 / k)\left(\lambda_{S_{1}} \lambda_{D_{G}}+\lambda_{S_{2}} \lambda_{D_{F}}\right)+\lambda_{m}\right]}=\theta_{1}, \\
& \lim _{k \rightarrow \infty} \frac{\rho\left[(1+1 / k) \lambda_{S_{2}} \lambda_{D_{F}}+\lambda_{m}\right]}{1-\rho\left[(1+1 / k)\left(\lambda_{S_{1}} \lambda_{D_{G}}+\lambda_{S_{2}} \lambda_{D_{F}}\right)+\lambda_{m}\right]}=\theta_{2},
\end{aligned}
$$

where $\theta_{1}=\left(1+\rho \lambda_{S_{1}} \lambda_{D_{G}}\right) /\left(1-\rho\left(\lambda_{S_{1}} \lambda_{D_{G}}+\lambda_{S_{2}} \lambda_{D_{F}}+\lambda_{m}\right)\right)$ and $\theta_{2}=\rho\left(\lambda_{S_{2}} \lambda_{D_{F}}+\lambda_{m}\right) /\left(1-\rho\left(\lambda_{S_{1}} \lambda_{D_{G}}+\right.\right.$ $\left.\left.\lambda_{S_{2}} \lambda_{D_{F}}+\lambda_{m}\right)\right)$.

Utilizing (2.40) and (2.44), we deduce from (2.39) that

$$
\begin{aligned}
\left\|z_{k+1}^{\prime}-z_{k}^{\prime}\right\| \leq & \left(\sqrt{1-2 \alpha+64 C \delta_{1}^{2}}+\sqrt{\frac{1+\rho(1+1 / k) \lambda_{S_{1}} \lambda_{D_{G}}}{1-\rho\left[(1+1 / k)\left(\lambda_{S_{1}} \lambda_{D_{G}}+\lambda_{S_{2}} \lambda_{D_{F}}\right)+\lambda_{m}\right]}}\right)\left\|x_{k}-x_{k-1}\right\| \\
& +\sqrt{\frac{\rho\left[(1+1 / k) \lambda_{S_{2}} \lambda_{D_{F}}+\lambda_{m}\right]}{1-\rho\left[(1+1 / k)\left(\lambda_{S_{1}} \lambda_{D_{G}}+\lambda_{S_{2}} \lambda_{D_{F}}\right)+\lambda_{m}\right]}}\left\|y_{k}-y_{k-1}\right\| \\
= & \left(B^{\prime}+\sqrt{\frac{1+\rho(1+1 / k) \lambda_{S_{1}} \lambda_{D_{G}}}{1-\rho\left[(1+1 / k)\left(\lambda_{S_{1}} \lambda_{D_{G}}+\lambda_{S_{2}} \lambda_{D_{F}}\right)+\lambda_{m}\right]}}\right)\left\|x_{k}-x_{k-1}\right\| \\
& +\sqrt{\frac{\rho\left[(1+1 / k) \lambda_{S_{2}} \lambda_{D_{F}}+\lambda_{m}\right]}{1-\rho\left[(1+1 / k)\left(\lambda_{S_{1}} \lambda_{D_{G}}+\lambda_{S_{2}} \lambda_{D_{F}}\right)+\lambda_{m}\right]}}\left\|y_{k}-y_{k-1}\right\|,
\end{aligned}
$$

where $B^{\prime}:=\sqrt{1-2 \alpha+64 C \delta_{1}^{2}}$. 
Journal of Applied Mathematics

On the other hand, again from Algorithm 2.3 we have

$$
\begin{aligned}
\left\|z_{k+1}^{\prime \prime}-z_{k}^{\prime \prime}\right\|= & \left\|g\left(y_{k}\right)-\gamma\left(T\left(u_{k}, t_{k}\right)-n\left(x_{k}\right)\right)-\left[g\left(y_{k-1}\right)-\gamma\left(T\left(u_{k-1}, t_{k-1}\right)-n\left(x_{k-1}\right)\right)\right]\right\| \\
\leq & \left\|y_{k}-y_{k-1}-\left(g\left(y_{k}\right)-g\left(y_{k-1}\right)\right)\right\| \\
& +\left\|y_{k}-y_{k-1}-\gamma\left[\left(T\left(u_{k}, t_{k}\right)-n\left(x_{k}\right)\right)-\left(T\left(u_{k-1}, t_{k-1}\right)-n\left(x_{k-1}\right)\right)\right]\right\| .
\end{aligned}
$$

Utilizing the same arguments as those for (2.40), we have

$$
\left\|y_{k}-y_{k-1}-\left(g\left(y_{k}\right)-g\left(y_{k-1}\right)\right)\right\|^{2} \leq\left(1-2 \beta+64 C_{2} \delta_{2}^{2}\right)\left\|y_{k}-y_{k-1}\right\|^{2}
$$

Since $T$ is Lipschitz continuous in both arguments, $H, V$ are $D$-Lipschitz continuous, and $n$ is Lipschitz continuous, we have

$$
\begin{aligned}
\left\|\left(T\left(u_{k}, t_{k}\right)-n\left(x_{k}\right)\right)-\left(T\left(u_{k-1}, t_{k-1}\right)-n\left(x_{k-1}\right)\right)\right\| \\
\quad=\left\|T\left(u_{k}, t_{k}\right)-T\left(u_{k-1}, t_{k}\right)+T\left(u_{k-1}, t_{k}\right)-T\left(u_{k-1}, t_{k-1}\right)-\left(n\left(x_{k}\right)-n\left(x_{k-1}\right)\right)\right\| \\
\quad \leq\left\|T\left(u_{k}, t_{k}\right)-T\left(u_{k-1}, t_{k}\right)\right\|+\left\|T\left(u_{k-1}, t_{k}\right)-T\left(u_{k-1}, t_{k-1}\right)\right\|+\left\|n\left(x_{k}\right)-n\left(x_{k-1}\right)\right\| \\
\quad \leq \lambda_{T_{1}}\left\|u_{k}-u_{k-1}\right\|+\lambda_{T_{2}}\left\|t_{k}-t_{k-1}\right\|+\lambda_{n}\left\|x_{k}-x_{k-1}\right\| \\
\quad \leq \lambda_{T_{1}}\left(1+\frac{1}{k}\right) D\left(H\left(x_{k}\right), H\left(x_{k-1}\right)\right)+\lambda_{T_{2}}\left(1+\frac{1}{k}\right) D\left(V\left(y_{k}\right), V\left(y_{k-1}\right)\right)+\lambda_{n}\left\|x_{k}-x_{k-1}\right\| \\
\quad \leq\left(1+\frac{1}{k}\right) \lambda_{T_{1}} \lambda_{D_{H}}\left\|x_{k}-x_{k-1}\right\|+\left(1+\frac{1}{k}\right) \lambda_{T_{2}} \lambda_{D_{V}}\left\|y_{k}-y_{k-1}\right\|+\lambda_{n}\left\|x_{k}-x_{k-1}\right\| \\
\quad=\left[\left(1+\frac{1}{k}\right) \lambda_{T_{1}} \lambda_{D_{H}}+\lambda_{n}\right]\left\|x_{k}-x_{k-1}\right\|+\left(1+\frac{1}{k}\right) \lambda_{T_{2}} \lambda_{D_{V}}\left\|y_{k}-y_{k-1}\right\| .
\end{aligned}
$$

Utilizing (2.49) and Proposition 1.1, we have

$$
\begin{aligned}
& \left\|y_{k}-y_{k-1}-\gamma\left[\left(T\left(u_{k}, t_{k}\right)-n\left(x_{k}\right)\right)-\left(T\left(u_{k-1}, t_{k-1}\right)-n\left(x_{k-1}\right)\right)\right]\right\|^{2} \\
& \leq\left\|y_{k}-y_{k-1}\right\|^{2}-2 \gamma\left\langle\left(T\left(u_{k}, t_{k}\right)-n\left(x_{k}\right)\right)-\left(T\left(u_{k-1}, t_{k-1}\right)-n\left(y_{k-1}\right)\right),\right. \\
& \left.J\left(y_{k}-y_{k-1}-\gamma\left[\left(T\left(u_{k}, t_{k}\right)-n\left(x_{k}\right)\right)-\left(T\left(u_{k-1}, t_{k-1}\right)-n\left(x_{k-1}\right)\right)\right]\right)\right\rangle \\
& \leq\left\|y_{k}-y_{k-1}\right\|^{2}+2 \gamma\left\|\left(T\left(u_{k}, t_{k}\right)-n\left(x_{k}\right)\right)-\left(T\left(u_{k-1}, t_{k-1}\right)-n\left(y_{k-1}\right)\right)\right\| \\
& \quad \times\left\|y_{k}-y_{k-1}-\gamma\left[\left(T\left(u_{k}, t_{k}\right)-n\left(x_{k}\right)\right)-\left(T\left(u_{k-1}, t_{k-1}\right)-n\left(x_{k-1}\right)\right)\right]\right\| \\
& \leq\left\|y_{k}-y_{k-1}\right\|^{2}+2 \gamma\left\{\left[\left(1+\frac{1}{k}\right) \lambda_{T_{1}} \lambda_{D_{H}}+\lambda_{n}\right]\left\|x_{k}-x_{k-1}\right\|+\left(1+\frac{1}{k}\right) \lambda_{T_{2}} \lambda_{D_{V}}\left\|y_{k}-y_{k-1}\right\|\right\} \\
& \times\left\|y_{k}-y_{k-1}-\gamma\left[\left(T\left(u_{k}, t_{k}\right)-n\left(x_{k}\right)\right)-\left(T\left(u_{k-1}, t_{k-1}\right)-n\left(x_{k-1}\right)\right)\right]\right\|
\end{aligned}
$$




$$
\begin{aligned}
\leq & \left\|y_{k}-y_{k-1}\right\|^{2}+r\left[\left(1+\frac{1}{k}\right) \lambda_{T_{1}} \lambda_{D_{H}}+\lambda_{n}\right] \\
& \times\left\{\left\|x_{k}-x_{k-1}\right\|^{2}+\left\|y_{k}-y_{k-1}-r\left[\left(T\left(u_{k}, t_{k}\right)-n\left(x_{k}\right)\right)-\left(T\left(u_{k-1}, t_{k-1}\right)-n\left(x_{k-1}\right)\right)\right]\right\|^{2}\right\} \\
& +\gamma\left(1+\frac{1}{k}\right) \lambda_{T_{2}} \lambda_{D_{V}} \\
& \times\left\{\left\|y_{k}-y_{k-1}\right\|^{2}+\left\|y_{k}-y_{k-1}-r\left[\left(T\left(u_{k}, t_{k}\right)-n\left(x_{k}\right)\right)-\left(T\left(u_{k-1}, t_{k-1}\right)-n\left(x_{k-1}\right)\right)\right]\right\|^{2}\right\} \\
= & {\left[1+\gamma\left(1+\frac{1}{k}\right) \lambda_{T_{2}} \lambda_{D_{V}}\right]\left\|y_{k}-y_{k-1}\right\|^{2}+r\left[\left(1+\frac{1}{k}\right)\left(\lambda_{T_{1}} \lambda_{D_{H}}+\lambda_{T_{2}} \lambda_{D_{V}}\right)+\lambda_{n}\right] } \\
& \times\left\|y_{k}-y_{k-1}-r\left[\left(T\left(u_{k}, t_{k}\right)-n\left(x_{k}\right)\right)-\left(T\left(u_{k-1}, t_{k-1}\right)-n\left(x_{k-1}\right)\right)\right]\right\|^{2} \\
& +\gamma\left[\left(1+\frac{1}{k}\right) \lambda_{T_{1}} \lambda_{D_{H}}+\lambda_{n}\right]\left\|x_{k}-x_{k-1}\right\|^{2},
\end{aligned}
$$

which implies that

$$
\begin{aligned}
& \left\|y_{k}-y_{k-1}-\gamma\left[\left(T\left(u_{k}, t_{k}\right)-n\left(x_{k}\right)\right)-\left(T\left(u_{k-1}, t_{k-1}\right)-n\left(x_{k-1}\right)\right)\right]\right\|^{2} \\
& \leq \frac{1+\gamma(1+1 / k) \lambda_{T_{2}} \lambda_{D_{V}}}{1-\gamma\left[(1+1 / k)\left(\lambda_{T_{1}} \lambda_{D_{H}}+\lambda_{T_{2}} \lambda_{D_{V}}\right)+\lambda_{n}\right]}\left\|y_{k}-y_{k-1}\right\|^{2} \\
& +\frac{\gamma\left[(1+1 / k) \lambda_{T_{1}} \lambda_{D_{H}}+\lambda_{n}\right]}{1-\gamma\left[(1+1 / k)\left(\lambda_{T_{1}} \lambda_{D_{H}}+\lambda_{T_{2}} \lambda_{D_{V}}\right)+\lambda_{n}\right]}\left\|x_{k}-x_{k-1}\right\|^{2} \\
& \leq \frac{1+\gamma(1+1 / k) \lambda_{T_{2}} \lambda_{D_{V}}}{1-\gamma\left[(1+1 / k)\left(\lambda_{T_{1}} \lambda_{D_{H}}+\lambda_{T_{2}} \lambda_{D_{V}}\right)+\lambda_{n}\right]}\left\|y_{k}-y_{k-1}\right\|^{2} \\
& +\frac{\gamma\left[(1+1 / k) \lambda_{T_{1}} \lambda_{D_{H}}+\lambda_{n}\right]}{1-\gamma\left[(1+1 / k)\left(\lambda_{T_{1}} \lambda_{D_{H}}+\lambda_{T_{2}} \lambda_{D_{V}}\right)+\lambda_{n}\right]}\left\|x_{k}-x_{k-1}\right\|^{2} \\
& +2 \sqrt{\frac{1+\gamma(1+1 / k) \lambda_{T_{2}} \lambda_{D_{V}}}{1-\gamma\left[(1+1 / k)\left(\lambda_{T_{1}} \lambda_{D_{H}}+\lambda_{T_{2}} \lambda_{D_{V}}\right)+\lambda_{n}\right]}} \sqrt{\frac{\gamma\left[(1+1 / k) \lambda_{T_{1}} \lambda_{D_{H}}+\lambda_{n}\right]}{1-\gamma\left[(1+1 / k)\left(\lambda_{T_{1}} \lambda_{D_{H}}+\lambda_{T_{2}} \lambda_{D_{V}}\right)+\lambda_{n}\right]}} \\
& \times\left\|y_{k}-y_{k-1}\right\|\left\|x_{k}-x_{k-1}\right\| \\
& =\left\{\sqrt{\frac{1+\gamma(1+1 / k) \lambda_{T_{2}} \lambda_{D_{V}}}{1-\gamma\left[(1+1 / k)\left(\lambda_{T_{1}} \lambda_{D_{H}}+\lambda_{T_{2}} \lambda_{D_{V}}\right)+\lambda_{n}\right]}}\left\|y_{k}-y_{k-1}\right\|\right. \\
& \left.+\sqrt{\frac{r\left[(1+1 / k) \lambda_{T_{1}} \lambda_{D_{H}}+\lambda_{n}\right]}{1-\gamma\left[(1+1 / k)\left(\lambda_{T_{1}} \lambda_{D_{H}}+\lambda_{T_{2}} \lambda_{D_{V}}\right)+\lambda_{n}\right]}}\left\|x_{k}-x_{k-1}\right\|\right\}^{2} .
\end{aligned}
$$


Thus, we have

$$
\begin{aligned}
&\left\|y_{k}-y_{k-1}-\gamma\left[\left(T\left(u_{k}, t_{k}\right)-n\left(x_{k}\right)\right)-\left(T\left(u_{k-1}, t_{k-1}\right)-n\left(x_{k-1}\right)\right)\right]\right\| \\
& \leq \sqrt{\frac{1+\gamma(1+1 / k) \lambda_{T_{2}} \lambda_{D_{V}}}{1-\gamma\left[(1+1 / k)\left(\lambda_{T_{1}} \lambda_{D_{H}}+\lambda_{T_{2}} \lambda_{D_{V}}\right)+\lambda_{n}\right]}}\left\|y_{k}-y_{k-1}\right\| \\
&+\sqrt{\frac{r\left[(1+1 / k) \lambda_{T_{1}} \lambda_{D_{H}}+\lambda_{n}\right]}{1-\gamma\left[(1+1 / k)\left(\lambda_{T_{1}} \lambda_{D_{H}}+\lambda_{T_{2}} \lambda_{D_{V}}\right)+\lambda_{n}\right]}}\left\|x_{k}-x_{k-1}\right\| .
\end{aligned}
$$

Note that

$$
\begin{aligned}
& \lim _{k \rightarrow \infty} \frac{1+\gamma(1+1 / k) \lambda_{T_{2}} \lambda_{D_{V}}}{1-\gamma\left[(1+1 / k)\left(\lambda_{T_{1}} \lambda_{D_{H}}+\lambda_{T_{2}} \lambda_{D_{V}}\right)+\lambda_{n}\right]}=\theta_{3}, \\
& \lim _{k \rightarrow \infty} \frac{\gamma\left[(1+1 / k) \lambda_{T_{1}} \lambda_{D_{H}}+\lambda_{n}\right]}{1-\gamma\left[(1+1 / k)\left(\lambda_{T_{1}} \lambda_{D_{H}}+\lambda_{T_{2}} \lambda_{D_{V}}\right)+\lambda_{n}\right]}=\theta_{4},
\end{aligned}
$$

where $\theta_{3}=\left(1+\gamma \lambda_{T_{2}} \lambda_{D_{V}}\right) /\left(1-\gamma\left(\lambda_{T_{1}} \lambda_{D_{H}}+\lambda_{T_{2}} \lambda_{D_{V}}+\lambda_{n}\right)\right)$ and $\theta_{4}=\gamma\left(\lambda_{T_{1}} \lambda_{D_{H}}+\lambda_{n}\right) /\left(1-\gamma\left(\lambda_{T_{1}} \lambda_{D_{H}}+\right.\right.$ $\left.\left.\lambda_{T_{2}} \lambda_{D_{V}}+\lambda_{n}\right)\right)$.

Utilizing (2.48) and (2.52), we deduce from (2.47) that

$$
\begin{aligned}
\left\|z_{k+1}^{\prime \prime}-z_{k}^{\prime \prime}\right\| \leq & \left(\sqrt{1-2 \beta+64 C \delta_{2}^{2}}+\sqrt{\frac{1+\gamma(1+1 / k) \lambda_{T_{2}} \lambda_{D_{V}}}{1-\gamma\left[(1+1 / k)\left(\lambda_{T_{1}} \lambda_{D_{H}}+\lambda_{T_{2}} \lambda_{D_{V}}\right)+\lambda_{n}\right]}}\right)\left\|y_{k}-y_{k-1}\right\| \\
& +\sqrt{\frac{\gamma\left[(1+1 / k) \lambda_{T_{1}} \lambda_{D_{H}}+\lambda_{n}\right]}{1-\gamma\left[(1+1 / k)\left(\lambda_{T_{1}} \lambda_{D_{H}}+\lambda_{T_{2}} \lambda_{D_{V}}\right)+\lambda_{n}\right]}}\left\|x_{k}-x_{k-1}\right\| \\
= & \left(B^{\prime \prime}+\sqrt{\frac{1+\gamma(1+1 / k) \lambda_{T_{2}} \lambda_{D_{V}}}{1-\gamma\left[(1+1 / k)\left(\lambda_{T_{1}} \lambda_{D_{H}}+\lambda_{T_{2}} \lambda_{D_{V}}\right)+\lambda_{n}\right]}}\right)\left\|y_{k}-y_{k-1}\right\| \\
& +\sqrt{\frac{\gamma\left[(1+1 / k) \lambda_{T_{1}} \lambda_{D_{H}}+\lambda_{n}\right]}{1-\gamma\left[(1+1 / k)\left(\lambda_{T_{1}} \lambda_{D_{H}}+\lambda_{T_{2}} \lambda_{D_{V}}\right)+\lambda_{n}\right]}}\left\|x_{k}-x_{k-1}\right\|,
\end{aligned}
$$

where $B^{\prime \prime}:=\sqrt{1-2 \beta+64 C \delta_{2}^{2}}$. 
Adding (2.46) and (2.54), we have

$$
\begin{aligned}
& \left\|z_{k+1}^{\prime}-z_{k}^{\prime}\right\|+\left\|z_{k+1}^{\prime \prime}-z_{k}^{\prime \prime}\right\| \\
& \leq\left(B^{\prime}+\sqrt{\frac{1+\rho(1+1 / k) \lambda_{S_{1}} \lambda_{D_{G}}}{1-\rho\left[(1+1 / k)\left(\lambda_{S_{1}} \lambda_{D_{G}}+\lambda_{S_{2}} \lambda_{D_{F}}\right)+\lambda_{m}\right]}}\right. \\
& \left.+\sqrt{\frac{\gamma\left[(1+1 / k) \lambda_{T_{1}} \lambda_{D_{H}}+\lambda_{n}\right]}{1-\gamma\left[(1+1 / k)\left(\lambda_{T_{1}} \lambda_{D_{H}}+\lambda_{T_{2}} \lambda_{D_{V}}\right)+\lambda_{n}\right]}}\right)\left\|x_{k}-x_{k-1}\right\| \\
& +\left(B^{\prime \prime}+\sqrt{\frac{\rho\left[(1+1 / k) \lambda_{S_{2}} \lambda_{D_{F}}+\lambda_{m}\right]}{1-\rho\left[(1+1 / k)\left(\lambda_{S_{1}} \lambda_{D_{G}}+\lambda_{S_{2}} \lambda_{D_{F}}\right)+\lambda_{m}\right]}}\right. \\
& \left.+\sqrt{\frac{1+\gamma(1+1 / k) \lambda_{T_{2}} \lambda_{D_{V}}}{1-\gamma\left[(1+1 / k)\left(\lambda_{T_{1}} \lambda_{D_{H}}+\lambda_{T_{2}} \lambda_{D_{V}}\right)+\lambda_{n}\right]}}\right)\left\|y_{k}-y_{k-1}\right\| .
\end{aligned}
$$

Also from (2.21), we have

$$
\begin{aligned}
\left\|x_{k}-x_{k-1}\right\| & =\left\|x_{k}-x_{k-1}-\left(f\left(x_{k}\right)-f\left(x_{k-1}\right)\right)+J_{M}^{\rho}\left(z_{k}^{\prime}\right)-J_{M}^{\rho}\left(z_{k-1}^{\prime}\right)\right\| \\
& \leq\left\|x_{k}-x_{k-1}-\left(f\left(x_{k}\right)-f\left(x_{k-1}\right)\right)\right\|+\left\|J_{M}^{\rho}\left(z_{k}^{\prime}\right)-J_{M}^{\rho}\left(z_{k-1}^{\prime}\right)\right\| \\
& \leq B^{\prime}\left\|x_{k}-x_{k-1}\right\|+\left\|z_{k}^{\prime}-z_{k-1}^{\prime}\right\|,
\end{aligned}
$$

which implies that

$$
\begin{aligned}
\left\|x_{k}-x_{k-1}\right\| & \leq \frac{1}{1-B^{\prime}}\left\|z_{k}^{\prime}-z_{k-1}^{\prime}\right\|, \\
\left\|y_{k}-y_{k-1}\right\| & =\left\|y_{k}-y_{k-1}-\left(g\left(y_{k}\right)-g\left(y_{k-1}\right)\right)+J_{N}^{\gamma}\left(z_{k}^{\prime \prime}\right)-J_{N}^{\gamma}\left(z_{k-1}^{\prime \prime}\right)\right\| \\
& \leq\left\|y_{k}-y_{k-1}-\left(g\left(y_{k}\right)-g\left(y_{k-1}\right)\right)\right\|+\left\|J_{N}^{\gamma}\left(z_{k}^{\prime \prime}\right)-J_{N}^{\gamma}\left(z_{k-1}^{\prime \prime}\right)\right\| \\
& \leq B^{\prime \prime}\left\|y_{k}-y_{k-1}\right\|+\left\|z_{k}^{\prime \prime}-z_{k-1}^{\prime \prime}\right\|,
\end{aligned}
$$

which implies that

$$
\left\|y_{k}-y_{k-1}\right\| \leq \frac{1}{1-B^{\prime \prime}}\left\|z_{k}^{\prime \prime}-z_{k-1}^{\prime \prime}\right\| .
$$


Utilizing (2.57) and (2.59), we conclude from (2.55) that

$$
\begin{aligned}
& \left\|z_{k+1}^{\prime}-z_{k}^{\prime}\right\|+\left\|z_{k+1}^{\prime \prime}-z_{k}^{\prime \prime}\right\| \\
& \leq\left\{B^{\prime}+\sqrt{\frac{1+\rho(1+1 / k) \lambda_{S_{1}} \lambda_{D_{G}}}{1-\rho\left[(1+1 / k)\left(\lambda_{S_{1}} \lambda_{D_{G}}+\lambda_{S_{2}} \lambda_{D_{F}}\right)+\lambda_{m}\right]}}\right. \\
& \left.+\sqrt{\frac{\gamma\left[(1+1 / k) \lambda_{T_{1}} \lambda_{D_{H}}+\lambda_{n}\right]}{1-\gamma\left[(1+1 / k)\left(\lambda_{T_{1}} \lambda_{D_{H}}+\lambda_{T_{2}} \lambda_{D_{V}}\right)+\lambda_{n}\right]}}\right\} \frac{1}{1-B^{\prime}}\left\|z_{k}^{\prime}-z_{k-1}^{\prime}\right\| \\
& +\left\{B^{\prime \prime}+\sqrt{\frac{\rho\left[(1+1 / k) \lambda_{S_{2}} \lambda_{D_{F}}+\lambda_{m}\right]}{1-\rho\left[(1+1 / k)\left(\lambda_{S_{1}} \lambda_{D_{G}}+\lambda_{S_{2}} \lambda_{D_{F}}\right)+\lambda_{m}\right]}}\right. \\
& \left.+\sqrt{\frac{1+\gamma(1+1 / k) \lambda_{T_{2}} \lambda_{D_{V}}}{1-\gamma\left[(1+1 / k)\left(\lambda_{T_{1}} \lambda_{D_{H}}+\lambda_{T_{2}} \lambda_{D_{V}}\right)+\lambda_{n}\right]}}\right\} \frac{1}{1-B^{\prime \prime}}\left\|z_{k}^{\prime \prime}-z_{k-1}^{\prime \prime}\right\| .
\end{aligned}
$$

Observe that

$$
\begin{aligned}
& \lim _{k \rightarrow \infty}\left\{B^{\prime}+\sqrt{\frac{1+\rho(1+1 / k) \lambda_{S_{1}} \lambda_{D_{G}}}{1-\rho\left[(1+1 / k)\left(\lambda_{S_{1}} \lambda_{D_{G}}+\lambda_{S_{2}} \lambda_{D_{F}}\right)+\lambda_{m}\right]}}\right. \\
& \left.\quad+\sqrt{\frac{r\left[(1+1 / k) \lambda_{T_{1}} \lambda_{D_{H}}+\lambda_{n}\right]}{1-\gamma\left[(1+1 / k)\left(\lambda_{T_{1}} \lambda_{D_{H}}+\lambda_{T_{2}} \lambda_{D_{V}}\right)+\lambda_{n}\right]}}\right\} \frac{1}{1-B^{\prime}}=\frac{B^{\prime}+\sqrt{\theta_{1}}+\sqrt{\theta_{4}}}{1-B^{\prime}} \leq \theta, \\
& \lim _{k \rightarrow \infty}\left\{B^{\prime \prime}+\sqrt{\frac{\rho\left[(1+1 / k) \lambda_{S_{2}} \lambda_{D_{F}}+\lambda_{m}\right]}{1-\rho\left[(1+1 / k)\left(\lambda_{S_{1}} \lambda_{D_{G}}+\lambda_{S_{2}} \lambda_{D_{F}}\right)+\lambda_{m}\right]}}\right. \\
& \left.+\sqrt{\frac{1+\gamma(1+1 / k) \lambda_{T_{2}} \lambda_{D_{V}}}{1-\gamma\left[(1+1 / k)\left(\lambda_{T_{1}} \lambda_{D_{H}}+\lambda_{T_{2}} \lambda_{D_{V}}\right)+\lambda_{n}\right]}}\right\} \frac{1}{1-B^{\prime \prime}}=\frac{B^{\prime \prime}+\sqrt{\theta_{2}}+\sqrt{\theta_{3}}}{1-B^{\prime \prime}} \leq \theta,
\end{aligned}
$$

where

$$
\theta=\max \left\{\frac{B^{\prime}+\sqrt{\theta_{1}}+\sqrt{\theta_{4}}}{1-B^{\prime}}, \frac{B^{\prime \prime}+\sqrt{\theta_{2}}+\sqrt{\theta_{3}}}{1-B^{\prime \prime}}\right\} .
$$


By (2.38), we know that $0<\theta<1$. Now take a fixed $\theta_{0} \in(\theta, 1)$ arbitrarily. Then from $(2.61)$ and (2.31) it follows that there exists an integer $\bar{k} \geq 1$ such that for all $k \geq \bar{k}$,

$$
\begin{aligned}
& \left\{B^{\prime}+\sqrt{\frac{1+\rho(1+1 / k) \lambda_{S_{1}} \lambda_{D_{G}}}{1-\rho\left[(1+1 / k)\left(\lambda_{S_{1}} \lambda_{D_{G}}+\lambda_{S_{2}} \lambda_{D_{F}}\right)+\lambda_{m}\right]}}\right. \\
& \left.\quad+\sqrt{\left.\frac{r\left[(1+1 / k) \lambda_{T_{1}} \lambda_{D_{H}}+\lambda_{n}\right]}{1-\gamma\left[(1+1 / k)\left(\lambda_{T_{1}} \lambda_{D_{H}}+\lambda_{T_{2}} \lambda_{D_{V}}\right)+\lambda_{n}\right]}\right\}}\right\} \frac{1}{1-B^{\prime}}<\theta_{0}, \\
& \left\{B^{\prime \prime}+\sqrt{\frac{\rho\left[(1+1 / k) \lambda_{S_{2}} \lambda_{D_{F}}+\lambda_{m}\right]}{1-\rho\left[(1+1 / k)\left(\lambda_{S_{1}} \lambda_{D_{G}}+\lambda_{S_{2}} \lambda_{D_{F}}\right)+\lambda_{m}\right]}}\right. \\
& \left.+\sqrt{\frac{1+\gamma(1+1 / k) \lambda_{T_{2}} \lambda_{D_{V}}}{1-\gamma\left[(1+1 / k)\left(\lambda_{T_{1}} \lambda_{D_{H}}+\lambda_{T_{2}} \lambda_{D_{V}}\right)+\lambda_{n}\right]}}\right\} \frac{1}{1-B^{\prime \prime}}<\theta_{0} .
\end{aligned}
$$

So, we obtain from (2.60) that

$$
\left\|z_{k+1}^{\prime}-z_{k}^{\prime}\right\|+\left\|z_{k+1}^{\prime \prime}-z_{k}^{\prime \prime}\right\| \leq \theta_{0}\left(\left\|z_{k}^{\prime}-z_{k-1}^{\prime}\right\|+\left\|z_{k}^{\prime \prime}-z_{k-1}^{\prime \prime}\right\|\right), \quad \forall k \geq \bar{k},
$$

which implies that $\left\{z_{k}^{\prime}\right\}$ and $\left\{z_{k}^{\prime \prime}\right\}$ are both Cauchy sequences. Thus, there exist $z^{\prime} \in E_{1}$ and $z^{\prime \prime} \in E_{2}$ such that $z_{k}^{\prime} \rightarrow z^{\prime}$ and $z_{k}^{\prime \prime} \rightarrow z^{\prime \prime}$ as $k \rightarrow \infty$. From (2.57) and (2.59) it follows that $\left\{x_{k}\right\}$ and $\left\{y_{k}\right\}$ are also Cauchy sequences in $E_{1}$ and $E_{2}$, respectively, that is, there exist $x \in E_{1}, y \in E_{2}$ such that $x_{k} \rightarrow x$ and $y_{k} \rightarrow y$ as $k \rightarrow \infty$.

Also from (2.22), we have

$$
\begin{aligned}
\left\|u_{k+1}-u_{k}\right\| & \leq\left(1+\frac{1}{k+1}\right) D\left(H\left(x_{k+1}\right), H\left(x_{k}\right)\right) \leq\left(1+\frac{1}{k+1}\right) \lambda_{D_{H}}\left\|x_{k+1}-x_{k}\right\| \\
\left\|v_{k+1}-v_{k}\right\| & \leq\left(1+\frac{1}{k+1}\right) D\left(F\left(y_{k+1}\right), F\left(y_{k}\right)\right) \leq\left(1+\frac{1}{k+1}\right) \lambda_{D_{F}}\left\|y_{k+1}-y_{k}\right\| \\
\left\|s_{k+1}-s_{k}\right\| & \leq\left(1+\frac{1}{k+1}\right) D\left(G\left(x_{k+1}\right), G\left(x_{k}\right)\right) \leq\left(1+\frac{1}{k+1}\right) \lambda_{D_{G}}\left\|x_{k+1}-x_{k}\right\| \\
\left\|t_{k+1}-t_{k}\right\| & \leq\left(1+\frac{1}{k+1}\right) D\left(V\left(y_{k+1}\right), V\left(y_{k}\right)\right) \leq\left(1+\frac{1}{k+1}\right) \lambda_{D_{V}}\left\|y_{k+1}-y_{k}\right\|
\end{aligned}
$$

and hence, $\left\{u_{k}\right\},\left\{v_{k}\right\},\left\{s_{k}\right\}$, and $\left\{t_{k}\right\}$ are also Cauchy sequences. Accordingly, there exist $u, s \in$ $E_{1}$ and $v, t \in E_{2}$ such that $u_{k} \rightarrow u, v_{k} \rightarrow v, s_{k} \rightarrow s$, and $t_{k} \rightarrow t$, respectively.

Now, we will show that $u \in H(x), v \in F(y), s \in G(x)$, and $t \in V(y)$. Indeed, since $u_{k} \in H\left(x_{k}\right)$ and

$$
\begin{aligned}
d\left(u_{k}, H(x)\right) & \leq \max \left\{d\left(u_{k}, H(x)\right), \sup _{w_{1} \in H(x)} d\left(H\left(x_{k}\right), w_{1}\right)\right\} \\
& \leq \max \left\{\sup _{w_{2} \in H\left(x_{k}\right)} d\left(w_{2}, H(x)\right), \sup _{w_{1} \in H(x)} d\left(H\left(x_{k}\right), w_{1}\right)\right\} \\
& =D\left(H\left(x_{k}\right), H(x)\right),
\end{aligned}
$$


we have

$$
\begin{aligned}
d(u, H(x)) & \leq\left\|u-u_{k}\right\|+d\left(u_{k}, H(x)\right) \\
& \leq\left\|u-u_{k}\right\|+D\left(H\left(x_{k}\right), H(x)\right) \\
& \leq\left\|u-u_{k}\right\|+\lambda_{D_{H}}\left\|x_{k}-x\right\| \rightarrow 0 \text { as } k \rightarrow \infty,
\end{aligned}
$$

which implies that $d(u, H(x))=0$. Taking into account that $H(x) \in C B\left(E_{1}\right)$, we deduce that $u \in H(x)$. Similarly, we can show that $v \in F(y), s \in G(x)$ and $t \in V(y)$. By the continuity of $f, g, m, n, G, F, H, V, S, T, J_{M}^{\rho}, J_{N}^{\gamma}$ and Algorithm 2.3, we have

$$
\begin{gathered}
z^{\prime}=f(x)-\rho(S(s, v)-m(y))=J_{M}^{\rho}\left(z^{\prime}\right)-\rho(S(s, v)-m(y)) \in E_{1} \\
z^{\prime \prime}=g(y)-\gamma(T(u, t)-n(x))=J_{N}^{\gamma}\left(z^{\prime \prime}\right)-\gamma(T(u, t)-n(x)) \in E_{2} .
\end{gathered}
$$

By Propositions 2.1 and 2.2, the required result follows.

\section{Acknowledgments}

The research of L.-C. Ceng was partially supported by the National Science Foundation of China (11071169), Innovation Program of Shanghai Municipal Education Commission (09ZZ133), and Leading Academic Discipline Project of Shanghai Normal University (DZL707). The research of C.-F. Wen was partially supported by a Grant from NSC 100-2115M-037-001.

\section{References}

[1] C. Baiocchi and A. Capelo, Variational and Quasivariational Inequalities: Applications to Free Boundary Problems, John Wiley \& Sons, New York, NY, USA, 1984.

[2] G. Isac, V. A. Bulavsky, and V. V. Kalashnikov, Complementarity, Equilibrium, Efficiency and Economics, vol. 63 of Nonconvex Optimization and its Applications, Kluwer Academic Publishers, Dordrecht, The Netherlands, 2002.

[3] D. Kinderlehrer and G. Stampacchia, An Introduction to Variational Inequalities and Their Applications, vol. 88 of Pure and Applied Mathematics, Academic Press, New York, NY, USA, 1980.

[4] I. Konnov, Combined Relaxation Methods for Variational Inequalities, vol. 495 of Lecture Notes in Economics and Mathematical Systems, Springer, Berlin, Germany, 2001.

[5] A. Nagurney, Network Economics: A Variational Inequality Approach, vol. 1 of Advances in Computational Economics, Kluwer Academic Publishers, Dordrecht, The Netherlands, 1993.

[6] P. D. Panagiotopoulos and G. E. Stavroulakis, "New types of variational principles based on the notion of quasidifferentiability," Acta Mechanica, vol. 94, no. 3-4, pp. 171-194, 1992.

[7] M. Patriksson, Nonlinear Programming and Variational Inequality Problems: A Unified Approach, vol. 23 of Applied Optimization, Kluwer Academic Publishers, Dordrecht, The Netherlands, 1999.

[8] A. H. Siddiqi and Q. H. Ansari, "An algorithm for a class of quasivariational inequalities," Journal of Mathematical Analysis and Applications, vol. 145, no. 2, pp. 413-418, 1990.

[9] A. Hassouni and A. Moudafi, "A perturbed algorithm for variational inclusions," Journal of Mathematical Analysis and Applications, vol. 185, no. 3, pp. 706-712, 1994.

[10] M. A. Noor and K. I. Noor, "Multivalued variational inequalities and resolvent equations," Mathematical and Computer Modelling, vol. 26, no. 7, pp. 109-121, 1997.

[11] R. U. Verma, "Projection methods, algorithms, and a new system of nonlinear variational inequalities," Computers \& Mathematics with Applications, vol. 41, no. 7-8, pp. 1025-1031, 2001.

[12] J.-S. Pang, "Asymmetric variational inequality problems over product sets: applications and iterative methods," Mathematical Programming, vol. 31, no. 2, pp. 206-219, 1985. 
[13] G. Cohen and F. Chaplais, "Nested monotony for variational inequalities over product of spaces and convergence of iterative algorithms," Journal of Optimization Theory and Applications, vol. 59, no. 3, pp. 369-390, 1988.

[14] M. Binachi, "Pseudo p-monotone operators and variational inequalities," Tech. Rep. 6, Instituto di Econometria e Matematica per Decisioni Economiche, Universita Cattolica del Sacro Cuore, Milan, Italy, 1993.

[15] Q. H. Ansari and J.-C. Yao, "A fixed point theorem and its applications to a system of variational inequalities," Bulletin of the Australian Mathematical Society, vol. 59, no. 3, pp. 433-442, 1999.

[16] R. P. Agarwal, N.-J. Huang, and M.-Y. Tan, "Sensitivity analysis for a new system of generalized nonlinear mixed quasi-variational inclusions," Applied Mathematics Letters, vol. 17, no. 3, pp. 345-352, 2004.

[17] J. Peng and D. Zhu, "A new system of generalized mixed quasi-variational inclusions with $(H, \eta)$ monotone operators," Journal of Mathematical Analysis and Applications, vol. 327, no. 1, pp. 175-187, 2007.

[18] H.-Y. Lan, J. H. Kim, and Y. J. Cho, "On a new system of nonlinear $A$-monotone multivalued variational inclusions," Journal of Mathematical Analysis and Applications, vol. 327, no. 1, pp. 481-493, 2007.

[19] R. Ahmad, Q. H. Ansari, and S. S. Irfan, "Generalized variational inclusions and generalized resolvent equations in Banach spaces," Computers \& Mathematics with Applications, vol. 49, no. 11-12, pp. 18251835, 2005.

[20] S. S. Chang, Y. J. Cho, B. S. Lee, and I. H. Jung, "Generalized set-valued variational inclusions in Banach spaces," Journal of Mathematical Analysis and Applications, vol. 246, no. 2, pp. 409-422, 2000.

[21] N.-J. Huang, "A new class of generalized set-valued implicit variational inclusions in Banach spaces with an application," Computers \& Mathematics with Applications, vol. 41, no. 7-8, pp. 937-943, 2001.

[22] L. C. Ceng, S. Schaible, and J. C. Yao, "On the characterization of strong convergence of an iterative algorithm for a class of multi-valued variational inclusions," Mathematical Methods of Operations Research, vol. 70, no. 1, pp. 1-12, 2009.

[23] L.-C. Zeng, S.-M. Guu, and J.-C. Yao, "Characterization of $H$-monotone operators with applications to variational inclusions," Computers \& Mathematics with Applications, vol. 50, no. 3-4, pp. 329-337, 2005.

[24] X. P. Ding, J.-C. Yao, and L.-C. Zeng, "Existence and algorithm of solutions for generalized strongly nonlinear mixed variational-like inequalities in Banach spaces," Computers $\mathcal{E}$ Mathematics with Applications, vol. 55, no. 4, pp. 669-679, 2008.

[25] L.-C. Ceng, S.-M. Guu, and J.-C. Yao, "Iterative approximation of solutions for a class of completely generalized set-valued quasi-variational inclusions," Computers $\mathcal{E}$ Mathematics with Applications, vol. 56, no. 4, pp. 978-987, 2008.

[26] L.-C. Zeng, S.-M. Guu, and J.-C. Yao, "Three-step iterative algorithms for solving the system of generalized mixed quasi-variational-like inclusions," Computers \& Mathematics with Applications, vol. 53, no. 10 , pp. $1572-1581,2007$.

[27] L.-C. Zeng, S.-M. Guu, and J.-C. Yao, "Iterative algorithm for completely generalized set-valued strongly nonlinear mixed variational-like inequalities," Computers \& Mathematics with Applications, vol. 50, no. 5-6, pp. 935-945, 2005.

[28] L.-C. Zeng, S.-M. Guu, and J.-C. Yao, "An iterative method for generalized nonlinear set-valued mixed quasi-variational inequalities with $H$-monotone mappings," Computers $\mathcal{E}$ Mathematics with Applications, vol. 54, no. 4, pp. 476-483, 2007.

[29] L.-C. Zeng, Q. H. Ansari, and J.-C. Yao, "General iterative algorithms for solving mixed quasivariational-like inclusions," Computers $\mathcal{E}$ Mathematics with Applications, vol. 56, no. 10, pp. 2455-2467, 2008.

[30] L.-C. Ceng, S.-M. Guu, and J.-C. Yao, "Iterative algorithm for finding approximate solutions of mixed quasi-variational-like inclusions," Computers \& Mathematics with Applications, vol. 56, no. 4, pp. 942 952, 2008.

[31] R. Ahmad and J. C. Yao, "System of generalized resolvent equations with corresponding system of variational inclusions," Journal of Global Optimization, vol. 44, no. 2, pp. 297-309, 2009.

[32] Ya. Alber and J.-C. Yao, "Algorithm for generalized multi-valued co-variational inequalities in Banach spaces," Functional Differential Equations, vol. 7, no. 1-2, pp. 5-13, 2000.

[33] Y. I. Alber, "Metric and generalized projection operators in Banach spaces: properties and applications," in Theory and Applications of Nonlinear Operators of Accretive and Monotone Type, vol. 178 of Lecture Notes in Pure and Applied Mathematics, pp. 15-50, Dekker, New York, NY, USA, 1996.

[34] S. B. Nadler Jr., "Multi-valued contraction mappings," Pacific Journal of Mathematics, vol. 30, pp. 475488, 1969. 


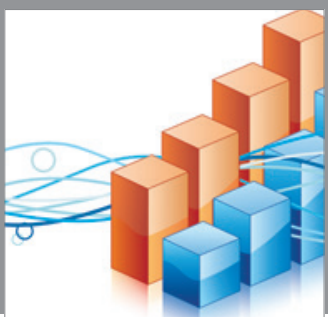

Advances in

Operations Research

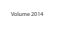

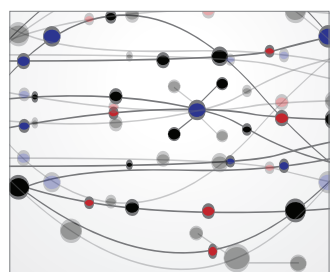

\section{The Scientific} World Journal
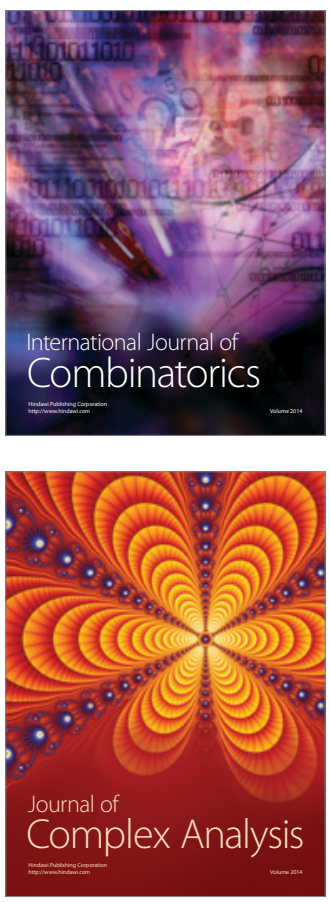

International Journal of

Mathematics and

Mathematical

Sciences
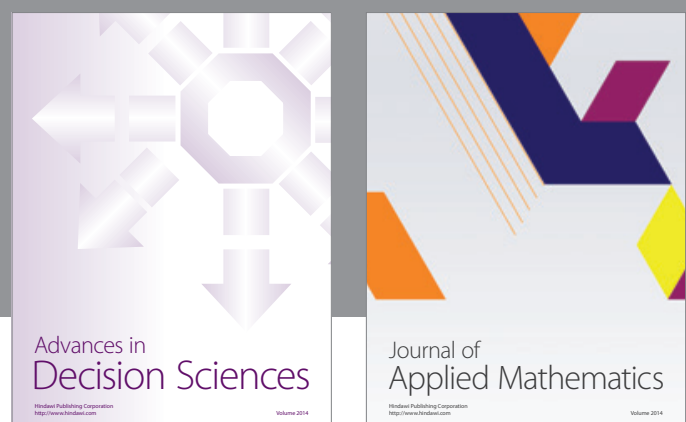

Journal of

Applied Mathematics
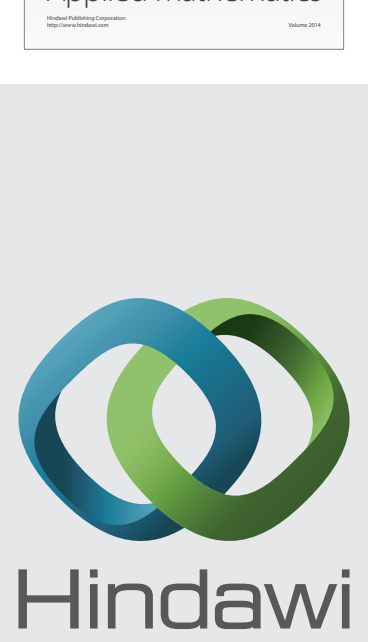

Submit your manuscripts at http://www.hindawi.com
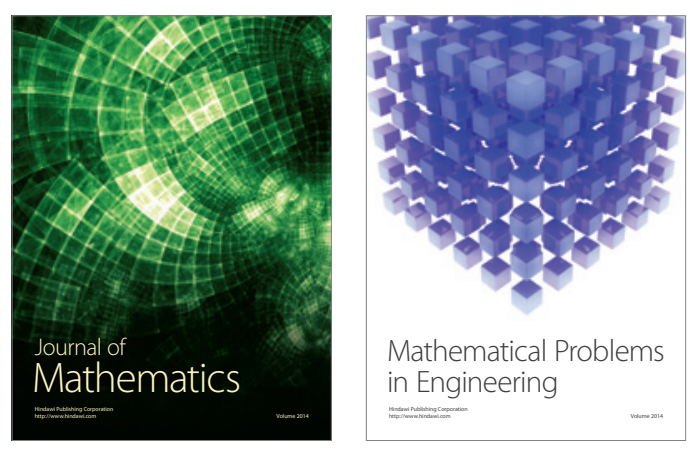

Mathematical Problems in Engineering
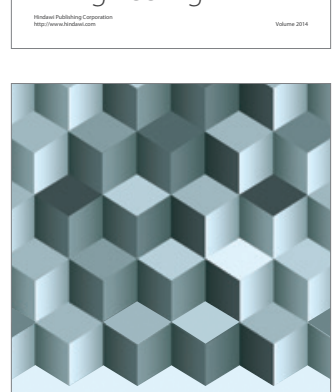

Journal of

Function Spaces
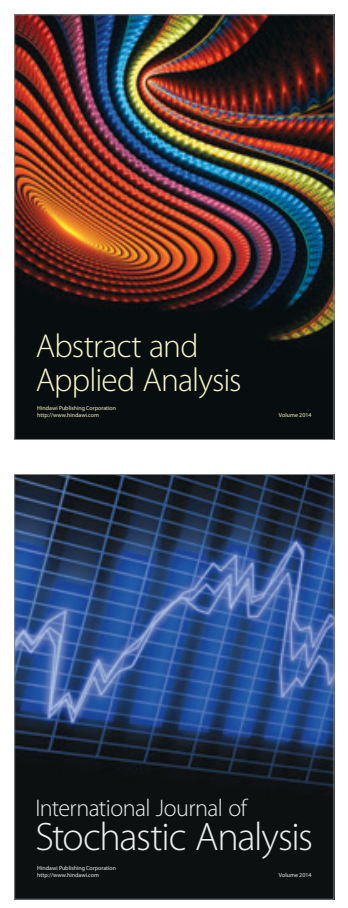

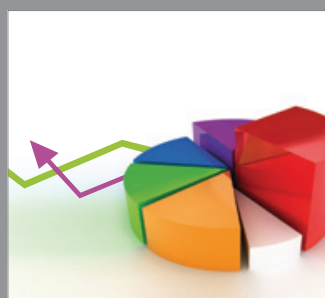

ournal of

Probability and Statistics

Promensencen
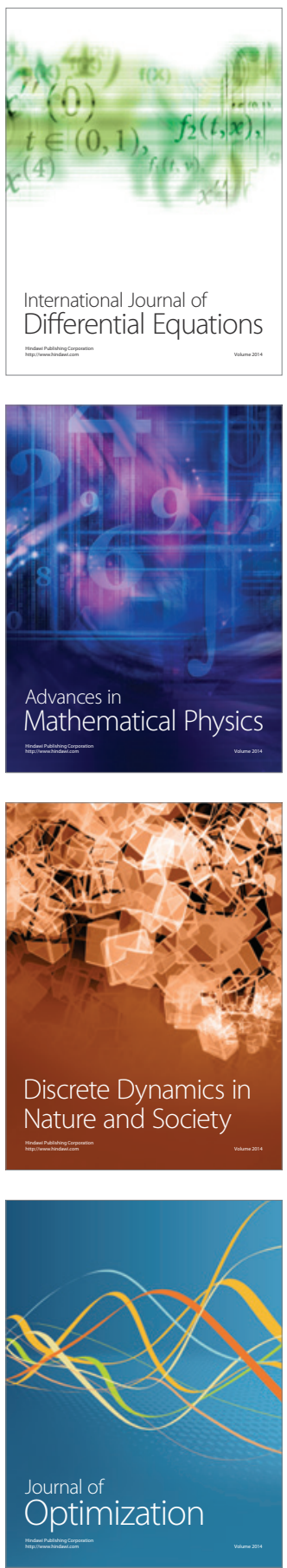